Encyclopedia of Thermal Stresses 


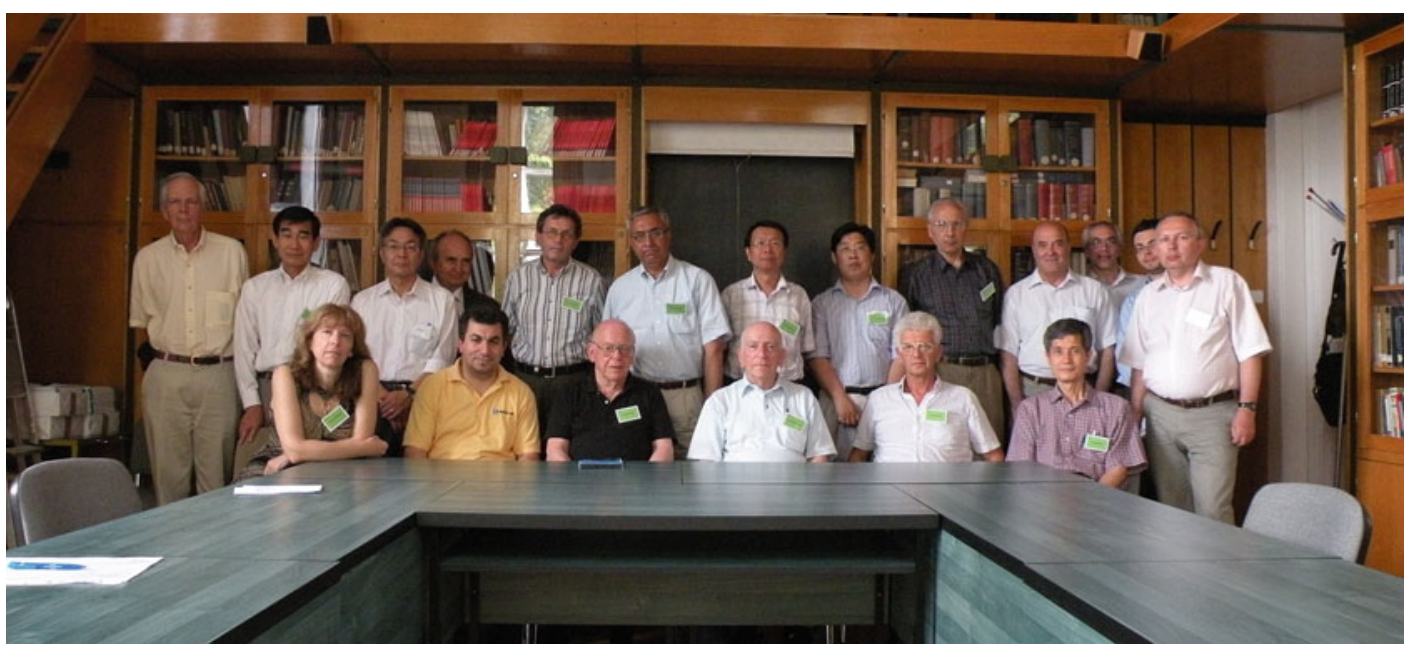

Meeting of Section Editors of the Encyclopedia of Thermal Stresses at the 9th International Congress on Thermal Stresses (ICTS) in Budapest on June 8, 2011.

Sitting, from left to right:

Marina V. Shitikova, Voronezh State University of Architecture and Civil Engineering,

Russia;

Piergiovanni Marzocca, Clarkson University, Potsdam, NY, U.S.A.;

Harry H. Hilton, University of Illinois at Urbana-Champaign, Urbana, IL, U.S.A.

Richard B. Hetnarski, Rochester Institute of Technology, U.S.A.; Editor-in-Chief of the ETS;

President of the ICTS organization; Co-Chair of the 9th Int. Congress on Thermal Stresses;

Andras Szekeres, Budapest University of Technology and Economics, Chair of the 9th

Int. Congress on Thermal Stresses;

Naotake Noda, University of Shizuoka, Hamamatsu, Japan; Co-Chair of the 9th Int.

Congress on Thermal Stresses.

Standing, from left to right:

Theodore R. Tauchert, University of Kentucky, U.S.A.; Secretary General of the ICTS

organization;

Fumihiro Ashida, Shimane University, Japan;

Sei Ueda, Osaka Institute of Technology, Japan;

Jan Taler, Cracow University of Technology, Poland;

Yuriy A. Rossikhin, Voronezh State University of Architecture and Civil Engineering, Russia;

M. Reza Eslami, Amirkabir University of Technology, Tehran, Iran;

Ching-Kong Chao, National Taiwan University of Science and Technology, Taipei, Taiwan,

Republic of China;

Cun-Fa Gao, Nanjing University of Aeronautics and Astronautics, People's Republic

of China;

Dorin Iesan, University of Iasi, Romania;

Stan Chirita, University of Iasi, Romania;

Vincenzo Tibullo and Vittorio Zampoli, representing Michele Ciarletta, DIIMA, University of Salerno, Italy;

Roman Kushnir, Pidstryhach Institute for Applied Problems of Mechanics and

Mathematics, National Academy of Sciences of Ukraine, Lviv, Ukraine.

(Photograph taken by Yuriy V. Tokovyy, Pidstryhach Institute for Applied Problems of

Mechanics and Mathematics, National Academy of Sciences of Ukraine, Lviv, Ukraine). 
Richard B. Hetnarski

Editor

\section{Encyclopedia of Thermal Stresses}

With 3310 Figures and 371 Tables

包 Springer Reference 


\section{Editor}

Professor Emeritus

Richard B. Hetnarski

Department of Mechanical Engineering

Rochester Institute of Technology

Rochester, NY, USA

and

Naples, FL, USA

ISBN 978-94-007-2738-0

ISBN 978-94-007-2739-7 (eBook)

ISBN Bundle 978-94-007-2740-3 (print and electronic bundle)

DOI 10.1007/978-94-007-2739-7

Springer Dordrecht Heidelberg New York London

Library of Congress Control Number: 2013951772

(C) Springer Science+Business Media Dordrecht 2014

This work is subject to copyright. All rights are reserved by the Publisher, whether the whole or part of the material is concerned, specifically the rights of translation, reprinting, reuse of illustrations, recitation, broadcasting, reproduction on microfilms or in any other physical way, and transmission or information storage and retrieval, electronic adaptation, computer software, or by similar or dissimilar methodology now known or hereafter developed. Exempted from this legal reservation are brief excerpts in connection with reviews or scholarly analysis or material supplied specifically for the purpose of being entered and executed on a computer system, for exclusive use by the purchaser of the work. Duplication of this publication or parts thereof is permitted only under the provisions of the Copyright Law of the Publisher's location, in its current version, and permission for use must always be obtained from Springer. Permissions for use may be obtained through RightsLink at the Copyright Clearance Center. Violations are liable to prosecution under the respective Copyright Law.

The use of general descriptive names, registered names, trademarks, service marks, etc. in this publication does not imply, even in the absence of a specific statement, that such names are exempt from the relevant protective laws and regulations and therefore free for general use.

While the advice and information in this book are believed to be true and accurate at the date of publication, neither the authors nor the editors nor the publisher can accept any legal responsibility for any errors or omissions that may be made. The publisher makes no warranty, express or implied, with respect to the material contained herein.

Printed on acid-free paper

Springer is part of Springer Science+Business Media (www.springer.com) 


\section{Preface}

Doctrina multiplex, veritas una.

The Encyclopedia of Thermal Stresses (ETS) is an interdisciplinary reference work. Its main emphasis is on topics in the field of Thermal Stresses, but it contains also entries on related topics, such as Theory of Elasticity, Heat Conduction, Thermodynamics, appropriate areas of Applied Mathematics, and topics on Numerical Methods. The ETS is aimed at both undergraduate and graduate students, researchers and engineers. The creation of such an extensive publication required the effort of the Editorial Board consisting of 29 section editors, experts in their fields of specialization, who represent 15 countries. The hard work of preparation of entries was accomplished by 614 authors, experts in the specific topics, representing 61 countries. The ETS is the largest single publication devoted to the field of Thermal Stresses ever published. It contains 11 volumes comprising altogether 6725 pages. Its creation took two-and-a-half years of hard work of devoted section editors, as well as the devoted authors of 708 entries.

The field of Mechanics called Thermoelasticity and Thermal Stresses was started with the publication, in the year 1837, of the paper by J.-M.-C. Duhamel, Second Mémoire sur les Phénomènes Thermo-Mé caniques. Since then, well over 60 published books have been devoted explicitly to this field. The first journal that covers exclusively the subject, the Journal of Thermal Stresses, initially a quarterly, and now a monthly, was started in 1978, and the first International Congress of Thermal Stresses (which at that time was called a Symposium), was held in Hamamatsu, Japan, in 1995. The largest publication on the subject before creation of the ETS, was a set of five volumes entitled Thermal Stresses, directed at researchers and engineers. That series, which appeared in the years 1986 to 1999, was edited by this editor and published by Elsevier and Lastran Corporation; it comprised 27 chapters and a total of 2129 printed pages on state-of-the-art level, prepared by specialists. The dates mentioned above were the milestones in the development of the field of Thermoelasticity and Thermal Stresses. Over the years, the number of papers on the subject that have appeared in the literature has continued to increase, and this growth is an indication of the substantial interest of researchers devoting their time to this field, and of the number of users in industry depending upon this large volume of knowledge.

The ETS contains much of our knowledge on Thermal Stresses, including also some textbook information. Thus, the ETS should be useful to readers 
with various needs and various levels of preparation. As for the exposition of the material, the effort was directed toward the creation of many entries that are possibly original and new, even if the material itself has been well known for a very long time, which is necessary in order for the ETS to contain also the standard textbook knowledge. But even such information is presented here in more original form, in more original context, and with original illustrations or examples, whenever possible. Also, the ETS contains historical notes, controversial ideas, directions of future research, and - in particular-new ideas, new methods of solutions, and new results. The choice of material in the ETS is geared toward topics containing important developments, with the intention of opening wide perspectives for further expansion of the field of Thermal Stresses. Such a mixture is what should make the ETS an especially attractive and precious publication. And the goal of the ETS is not only to inform but also to encourage the young generation to work in the areas of science covered by the ETS.

For the authors, taking part in the creation of the ETS was considered both a privilege and hard work. All involved made an effort to do everything possible to arrive at the best result, as its effects hopefully will be felt for many years. The best specialists were invited to contribute entries. If some of those invited refused to participate, other experts were invited as replacements; however, at the end, not all planned entries found appropriate authors and some entries had to be abandoned.

Many of the authors worked in a close circle of similarly "initiated" colleagues, and if not for the opportunity to write entries for the ETS, they would not come "in the open" and would never write to the wider audience. Offering invitations and receiving acceptance of such invitations by so large a group of specialists was an ambitious effort. As a result, those who use the ETS should consider this publication not only as a source of information, but also as a source of intellectual satisfaction.

Special thanks are extended to Mr. Kevin J. Bradley, President - US Journals, at Taylor \& Francis, who was so kind as to offer his approval of granting copyright permissions, without charge, to use the contents of 40 papers published in the Journal of Thermal Stresses by their authors in the preparation of the ETS entries. The friendly attitude and generosity of Mr. Bradley contributed to the enrichment of the ETS with the results that otherwise would not be a part of it.

As editor, I express my congratulations and thanks to all who were onboard while the work on the ETS progressed: to the section editors and to the authors, whose names and affiliations may be found on pages that follow. My special thanks are extended to important ladies at Springer: Nathalie Jacobs and Lydia Mueller, who approached me in the spring of 2010 with the offer to undertake the work on the ETS, and to Editors Jutta JaegerHamers, Daniela Graf, and Gabriele Stjepanovic who worked so hard on leading the project from its inception to its conclusion. 


\section{Editor}

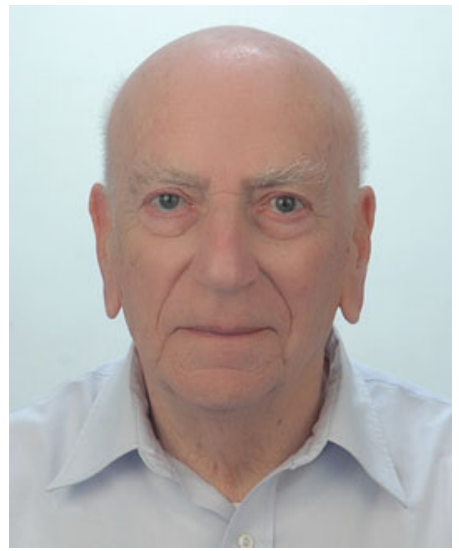

Richard B. Hetnarski Professor Emeritus, Department of Mechanical Engineering, Rochester Institute of Technology, Rochester, NY, USA

Naples, FL, USA 



\section{Section Editors}

Fumihiro Ashida Shimane University, Shimane, Japan

James Richard Barber University of Michigan, Ann Arbor, MI, USA

Janice Barton University of Southampton, Southampton, UK

Erasmo Carrera Politecnico di Torino, Torino, Italy

Ching-Kong Chao National Taiwan University of Science and Technology, Taipei, Taiwan, Republic of China

Stan Chirita “A1. I. Cuza” University of Iasi, Iasi, Romania

Michele Ciarletta DIIMA, University of Salerno, Fisciano (SA), Italy

M. Reza Eslami Amirkabir University of Technology, Tehran, Iran

Cun-Fa Gao Nanjing University of Aeronautics and Astronautics, Nanjing, People's Republic of China

Harry H. Hilton University of Illinois at Urbana-Champaign, Urbana, IL, USA

Dorin Iesan “A1. I. Cuza” University of Iasi, Iasi, Romania

Tatsuo Inoue Fukuyama University, Hiroshima, Japan

Roman Kushnir Pidstryhach Institute for Applied Problems of Mechanics and Mathematics, National Academy of Sciences of Ukraine, Lviv, Ukraine

Anatoly G. Lanin Institute of Association "LUCH", Podolsk, Russia

Lars-Erik Lindgren Luleå University of Technology, Luleå, Sweden

Piergiovanni Marzocca Clarkson University, Potsdam, NY, USA

Gerard Maugin Universite Pierre et Marie Curie, Paris, France

Roderick Melnik University of Waterloo, Waterloo, ON, Canada

Angelo Morro University of Genoa, Genoa, Italy

Naotake Noda University of Shizuoka, Hamamatsu, Japan

Hany Sherief University of Alexandria, Alexandria, Egypt 
Ephraim Suhir University of California at Santa Cruz, Santa Cruz, CA, USA

Jan Taler Cracow University of Technology, Cracow, Poland

Kumar Tamma University of Minnesota, Minneapolis, MN, USA

Theodore R. Tauchert University of Kentucky, Lexington, KY, USA

Yuriy V. Tokovyy Pidstryhach Institute for Applied Problems of Mechanics and Mathematics, National Academy of Sciences of Ukraine, Lviv, Ukraine National Taiwan University, Taipei, Taiwan, Republic of China

Andrzej Tylikowski Warsaw University of Technology, Warsaw, Poland

Sei Ueda Osaka Institute of Technology, Osaka, Japan

Xinwei Wang Iowa State University, Ames, IA, USA 


\section{Contributors}

Laith K. Abbas Institute of Launch Dynamics, Nanjing University of Sciences and Technology, Nanjing, People's Republic of China

Aerothermoelastic Behavior of Flat and Curved Panels

Aerothermoelastic Behavior of Lifting Surfaces

Aerothermoelastic Control of Lifting Surfaces

A. M. Abd El-Latief Department of Mathematics, Faculty of Science, University of Alexandria, Alexandria, Egypt

Boundary Element Method in Generalized Thermoelasticity

Fractional-Order Theory of Thermoelasticity

State-Space Approach to Generalized Thermoelasticity

Hillar Aben Institute of Cybernetics, Tallinn University of Technology, Tallinn, Estonia

Photoelasticity for the Measurement of Thermal Residual Stresses in Glass

Sayed M. Abo-Dahab Department of Mathematics, South Valley University, Qena, Egypt

Department of Mathematics, Taif University, Taif, Saudi Arabia

Surface Waves in Coupled and Generalized Thermoelasticity

Moustafa Saber Abou-Dina Department of Mathematics, Faculty of Science, Cairo University, Cairo, Giza, Egypt

Boundary Integral Formulation of the Plane Problems of Thermoelastostatics

Sarp Adali School of Engineering, University of KwaZulu-Natal, Durban, South Africa

Piezoelectric Actuation: Control of Linear Thermal Vibrations

Luciano Afferrante DMMM Politecnico di Bari, Bari, Italy

Thermoelastic Dynamic Instability (TEDI)

Carlos Agelet de Saracibar ETS Ingenieros de Caminos, Canales y Puertos, Universidad Politécnica de Cataluña, Barcelona Tech, Barcelona, Spain

International Center for Numerical Methods in Engineering (CIMNE), Barcelona, Spain

Shaped Metal Deposition Processes 
Esteban F. Aglietti Centro de Tecnología de Recursos Minerales y Cerámica (CETMIC), (CIC-CONICET-CCT La Plata), Buenos Aires, Argentina

Facultad de Ciencias Exactas - Universidad Nacional de La Plata, UNLP, Argentina

CONICET, La Plata, Argentina

Thermal Shock Resistance (TSR) and Thermal Fatigue Resistance (TFR) of Refractory Materials. Evaluation Method Based on the Dynamic Elastic Modulus

Mountajab Al-Hasan Department of Mathematics, Al-Baath University, Homs, Syrian Arab Republic

Coupled Dynamic Micropolar Problems of Thermoelasticity:

Stress-Temperature Equations of Motion of Ignaczak Type

Akbar Alibeigloo Department of Mechanical Engineering, Faculty of Engineering, Tarbiat Modares University, Tehran, Iran

Thermoelastic Behavior of FGM Smart Structures such as Plates and Cylindrical Panels

Thermoelasticity Analysis of Functionally Graded Beam with Integrated Surface Piezoelectric Layers

Three-Dimensional Semi-analytical Thermoelasticity Solution for a Functionally Graded Solid and an Annular Circular Plate

Mohammad A. Al-Nimr Jordan University of Science and Technology, Irbid, Jordan

The Royal Scientific Society (RSS), Amman, Jordan

Thermal Stress Under the Effect of Nonconventional Heat Conduction Models

Gülay Altay Department of Civil Engineering, Faculty of Engineering, Boğaziçi University, Istanbul, Turkey

Piezothermoelasticity with Hygro-Effects: Fundamental Theory

Piezothermoelasticity: Fundamental Theory

Piezothermoelasticity: Variational Principles

Variational Principles in Coupled Thermoelasticity

Bahadir Alyavuz Department of Civil Engineering, Gazi University, Ankara, Turkey

Propagation of Shock Waves in Thermoelastic Solids in View of Singular Surfaces

Christianne V. D. R. Anderson Department of Mechanical Engineering, University of Minnesota, Minneapolis, MN, USA

C- and F-Processes Model and Dynamic Thermoelasticity

Lars-Erik Andersson Department of Mathematics, Linköping University, Linköping, Sweden

Existence and Uniqueness for Thermoelastic Contact 
Nikolaos K. Anifantis Department of Mechanical Engineering and Aeronautics, University of Patras, Patras, Greece

\section{Crack Closure}

Reza Ansari Department of Mechanical Engineering, University of Guilan, Rasht, Iran

\section{Thermal Buckling of Carbon Nanotubes}

Johan Anton Institute of Cybernetics, Tallinn University of Technology, Tallinn, Estonia

Photoelasticity for the Measurement of Thermal Residual Stresses in Glass

Maksim Antonov Department of Materials Engineering, Tallinn University of Technology, Tallinn, Estonia

\section{Thermal Shock Resistance of Chromium Carbide-Based Cermets}

Moncef Aouadi Department of Mathematics and Computer Science, Institut Supérieur des Sciences Appliquées et de Technologie de Mateur, Université de Carthage, Tunisia

Classic and Generalized Thermoelastic Diffusion Theories

Fumihiro Ashida Department of Mechanical, Electrical, and Electronic Engineering, Interdisciplinary Graduate School of Science and Engineering, Shimane University, Matsue, Shimane, Japan

Piezoelectric Actuation: Adaptive Control of Thermal Displacement Piezoelectric Actuation: Control of Linear Thermal Displacement Piezoelectric Actuation: Control of Thermal Stress

Piezothermoelastic Analysis: Potential Function Method Piezothermoelastic Problems: Inverse Static and Quasi-Static Analyses

Ara Avetisyan Institute of Mechanics National Academy of Science of Armenia, Yerevan, Armenia

Magneto-thermo-elastic Equations of Thin Electroconductive Isotropic Shells and Plates: Linear Equations

Magneto-Thermoelastic Equations of Thin Electroconductive Isotropic Shells and Plates: Nonlinear Equations

Metin Aydogdu Department of Mechanical Engineering, Trakya University Faculty of Engineering and Architecture, Edirne, Turkey

Thermal Buckling of Composite Beam

S. Azevedo Department of Physics, School of Sciences, University of Minho, Guimarães, Portugal

Residual Stresses in Thin Films Evaluated by Different Experimental Techniques

Janusz Badur Energy Conversion Department, Institute of Fluid Flow Machinery, Polish Academy of Sciences, Gdańsk, Poland

Energy

Entropy: The Classical View

Thermodynamics of Thermo-deformable Solids 
Gevorg Y. Baghdasaryan Institute of Mechanics, National Academy of Sciences of Armenia, Yerevan, Armenia

Department of Informatics and Applied Mathematics, Yerevan State University, Yerevan, Armenia

Dynamic Stability of Electroconductive Cylindrical Shells in Magnetic Field

Ferromagnetic Plates and Shells

Thermoelastic Stability of Panels in High-Speed Flows: Linear Analysis

Thermoelastic Stability of Panels in High-Speed Flows: Nonlinear Analysis

Vibrations of Electroconductive Cylindrical Shells in a Magnetic Field

Leon Y. Bahar Department of Mechanical Engineering and Mechanics, Drexel University, Philadelphia, PA, USA

Direct Approach to Thermoelasticity

State-Space Approach to Thermoelasticity

Harvey E. Bair Bell Laboratories/Consultant, Newton, NJ, USA

Thermal Behavior of Thermoset Molding Compounds Used to Encapsulate IC Devices

Jerzy Banaszek Institute of Heat Engineering, Faculty of Power and Aeronautical Engineering, Warsaw University of Technology, Warsaw, Poland Phase Change Heat Transfer Problems

M. V. Banyas Timoshenko Institute of Mechanics, National Academy of Sciences of Ukraine, Kiev, Ukraine

Modeling of Thermomechanical Process in Growing Viscoplastic Bodies with Accounting of Microstructural Transformation

James R. Barber Department of Mechanical Engineering, University of Michigan, Ann Arbor, MI, USA

Contact Boundary Conditions

Frictionally Excited Thermoelastic Instability (TEI)

Swantje Bargmann Hamburg University of Technology, Institute of Continuum Mechanics and Material Mechanics, Hamburg, Germany

Helmholtz-Zentrum Geesthacht, Institute of Materials Research, Geesthacht, Germany

Second Sound Waves in Solids

Annalisa Barretta Department of Structures for Engineering and Architecture, University of Naples Federico II, Naples, Italy

Orthotropic Saint-Venant Beam Theory

Raffaele Barretta Department of Structures for Engineering and Architecture, University of Naples Federico II, Naples, Italy

Orthotropic Saint-Venant Beam Theory

Alberto Barroso Group of Elasticity and Strength of Materials, School of Engineering, University of Seville, Seville, Spain

Thermal Stresses in Composite Joints 
Romesh C. Batra Department of Engineering Science and Mechanics, Virginia Polytechnic Institute and State University, Blacksburg, VA, USA Thermal Shock Resistance of Functionally Graded Materials Thermally Induced Vibration, Circular Plates

Laurent Bechou Bordeaux University, Bordeaux, France

Thermal Stresses in a Tri-Material Assembly with Application to SiliconBased Photovoltaic Module (PVM)

Adib A. Becker Department of Mechanical, Materials and Manufacturing Engineering, University of Nottingham, Nottingham, UK

Finite Element Simulation of the Fusion Welding of Metal Components Including Post-weld Heat Treatment

A. Alibeygi Beni Department of Civil Engineering, Ramhormoz Branch, Islamic Azad University, Ramhormoz, Iran

Thermal Buckling Analysis of Orthotropic Nanoplates on Nonlinear Elastic Foundation

Rasajit K. Bera Department of Mathematics, Heritage Institute of Technology, East Kolkata, West Bengal, India

Thermally Induced Vibration, Elliptic Plates

Jean Michel Bergheau Université de Lyon, ENISE, LTDS, UMR 5513 CNRS, Saint-Etienne, France

Modeling and Numerical Simulation of Resistance Spot Welding Process

Pardeep K. Bhatti Intel Corporation, Chandler, AZ, USA

Thermal Stresses in Flip Chip BGA Packaging

Ryszard A. Białecki Institute of Thermal Technology, Silesian University of Technology, Gliwice, Poland

Green's Functions in Transient Heat Conduction

Victor Birman Engineering Education Center, Missouri University of Science and Technology, St. Louis, MO, USA

Functionally Graded Materials and Structures

Modeling and Analysis of Functionally Graded Materials and Structures

Paritosh Biswas Von Karman Society, Jalpaiguri, India

Plates on Elastic Foundation

Shells, Elastic Foundation

Thermally Induced Vibration, Laminated Plates

Thermally Induced Vibration, Orthotropic Plates

James P. Blanchard University of Wisconsin - Madison, Madison, WI, USA

Thermal Stresses in Solid-Wall Laser IFE Chambers

George E. Blandford Department of Civil Engineering, University of Kentucky, Lexington, KY, USA

Piezothermoelastic Analysis: Plate Theories 
James F. Blowey Department of Mathematical Sciences, Science Laboratories, Durham University, Durham, UK

Some Remarks on Functional Analysis

Dino Norberto Boccaccini Dipartimento di Ingegneria "Enzo Ferrari", Università degli Studi di Modena e Reggio Emilia, Modena, Italy

DTU Energy Conversion, Department of Energy Conversion and Storage, Technical University of Denmark, Roskilde, Denmark

Methods for Service Life Prediction in Refractory Materials

Microstructural Tailoring of Thermal Shock Resistance in Refractory

Materials

New Methods for the Assessment of Thermal Shock Resistance in

Refractory Materials

Aldo R. Boccaccini Department of Materials Science and Engineering, University of Erlangen-Nuremberg, Erlangen, Germany

Methods for Service Life Prediction in Refractory Materials

Yurii Aleksandrovitch Bogan Department of Deformable Solid Body,

M.A. Lavrentiev Institute of Hydrodynamics, Novosibirsk, Russia

Boundary Value Problems in Two-Dimensional Elastostatics of Anisotropic Solids

Two-Dimensional Problems in Thermo-elastostatics of Anisotropic Solids

Sami El Borgi Applied Mechanics and Systems Research Laboratory, Tunisia Polytechnic School, University of Carthage, La Marsa, Tunisia

Texas A\&M University at Qatar, Engineering Building, Mechanical Engineering Program, Education City, Doha, Qatar

Singularities of the Thermo-Magneto-Electro-Elastic Fields

Guido Borino Dipartimento di Ingegneria Civile, Ambientale, Aerospaziale e Materiali, Università di Palermo, Palermo, Italy

Nonlocal Elastic-Damage Models

Shakedown Under Thermomechanical Loads

Mark Andrew Bradford Centre for Infrastructure Engineering and Safety, School of Civil and Environmental Engineering, University of New South Wales, Sydney, NSW, Australia

Local Thermal Stability of Flanges and Webs

Shallow Arches: Thermal Buckling

Thermal Flexural-Torsional Stability of Arches

Salvatore Brischetto Department of Mechanical and Aerospace Engineering, Politecnico di Torino, Torino, Italy

Classical Governing Equations for the Thermomechanical Analysis of Shells

Constitutive and Geometrical Equations for the Thermomechanical Analysis of Shells

Refined and Advanced Governing Equations for the Thermomechanical Analysis of Shells 
Temperature Profiles in Composite and Sandwich Shells

Temperature Profiles in One-Layered and Multilayered Isotropic Shells

Thermal Stress Analysis of F unctionally Graded Material Plates

Thermomechanical Bending in Functionally Graded Material Shells

Thermomechanical Coupling in Multilayered Plates and Shells

Thermomechanical Coupling in Plate and Shell Structures - Some

Significant Results

Louis Brock Department of Mechanical Engineering, University of Kentucky, Lexington, KY, USA

Rapid Sliding Contact: Elastodynamic Steady State

Mircea Bîrsan Department of Mathematics, University “A.I. Cuza” of Iaşi, Iaşi, Romania

Faculty of Mathematics, University Duisburg-Essen, Essen, Germany

Cylindrical Elastic Bodies with Directors, Thermal Stresses

Cylindrical Orthotropic Thermoelastic Shells Modeled by Direct Approach Saint-Venant's Problem for Cosserat Elastic Shells

Emilian Bulgariu Department of Mathematics, Alexandra Ioan Cuza University of Iaşi, Iaşi, Romania

The University of Agricultural Sciences and Veterinary Medicine, Iaşi, Romania

Backward in Time Problems

Nonstandard Problems

Zbigniew Buliński Institute of Thermal Technology, Silesian University of Technology, Gliwice, Poland

Green's Functions in Transient Heat Conduction

Yaroslav Burak Pidstryhach Institute for Applied Problems of Mechanics and Mathematics, National Academy of Sciences of Ukraine, Lviv, Ukraine Local Gradient Thermomechanics

Dariusz Butrymowicz Institute of Fluid-Flow Machinery, Polish Academy of Sciences, Gdańsk, Poland

Thermodynamics of Machines

Francisca G. Caballero National Center for Metallurgical Research (CENIM-CSIC), Madrid, Spain

Microstructure Evolution in Steels

Marko Canađija Department of Engineering Mechanics, Faculty of Engineering, University of Rijeka, Rijeka, Croatia

Creep Analysis

Temperature-Dependent Thermoplasticity at Finite Strains

Rob N. Candler Los Angeles Electrical Engineering Department, University of California, LA, USA

Theory and Engineering of Micro-scale Resonators with Low

Thermoelastic Dissipation 
Maria Cannio Dipartimento di Ingegneria "Enzo Ferrari”, Università degli

Studi di Modena e Reggio Emilia, Modena, Italy

Methods for Service Life Prediction in Refractory Materials

Microstructural Tailoring of Thermal Shock Resistance in Refractory

Materials

New Methods for the Assessment of Thermal Shock Resistance in Refractory Materials

Sandra Carillo Dipartimento di Scienze di Base e Applicate per l'Ingegneria - Sezione Matematica, Sapienza Università di Roma, Rome, Italy

Fourier Series

Joaquim O. Carneiro Department of Physics, School of Sciences, University of Minho, Guimarães, Portugal

Residual Stresses in Thin Films Evaluated by Different Experimental Techniques

Erasmo Carrera Department of Mechanical and Aerospace Engineering, Politecnico di Torino, Torino, Italy

Classical Governing Equations for the Thermomechanical Analysis of

Shells

Constitutive and Geometrical Equations for the Thermomechanical Analysis of Shells

Refined and Advanced Governing Equations for the Thermomechanical Analysis of Shells

Temperature Profiles in Composite and Sandwich Shells

Temperature Profiles in One-Layered and Multilayered Isotropic Shells

Thermal Stress Analysis of Functionally Graded Material Plates

Thermomechanical Bending in Functionally Graded Material Shells

Thermomechanical Coupling in Multilayered Plates and Shells

Thermomechanical Coupling in Plate and Shell Structures - Some

Significant Results

Artur Cebula Institute of Thermal Power Engineering, Faculty of Mechanical Engineering, Cracow University of Technology, Cracow, Poland

Finite Volume Method in Heat Conduction

One-Dimensional Transient Heat Conduction in Plane Wall

One-Dimensional Transient Heat Conduction in Solid Cylinder

Miguel Cervera ETS Ingenieros de Caminos, Canales y Puertos, Universidad Politécnica de Cataluña, Barcelona Tech, Barcelona, Spain

International Center for Numerical Methods in Engineering (CIMNE), Barcelona, Spain

Shaped Metal Deposition Processes

Jean-Louis Chaboche Onera DMSM, The French Aerospace Lab, Châtillon, France

Modeling of Temperature Effects and Microstructural Evolutions in Plasticity 
Mama Chacha Faculty of Engineering and Applied Sciences, ALHOSN University, Abu Dhabi, United Arab Emirates

Porous Thermoelasticity with Applications

Rwei-Ching Chang Department of Mechanical and Computer-Aided Engineering, St. John's University, Tamsui, Taiwan, Republic of China Thermal Stresses of Thin Films on Flexible Substrates

Ching-Kong Chao Department of Mechanical Engineering, National Taiwan University of Science and Technology, Taipei, Taiwan, Republic of China

Annular Problems with a Point Heat Source

Bonded Circular Inclusions in Plane Thermoelasticity

Boundary Integral Equations for Notch Problems

Contact Stresses in an Infinite Plate with a Rigid Circular Inclusion

Creep Crack Growth

Curvilinear Cracks

Elliptic Inclusion in an Anisotropic Body

Heat Conduction Problems with Interacting Circular Inclusions

Heat Conduction Problems with Multiple Inclusions

Perturbation Solutions of Temperature Distribution of a Plane with a Nearly Circular Inclusion

Krishan K. Chawla Department of Materials Science and Engineering, University of Alabama at Birmingham, Birmingham, AL, USA

Thermal Shock Behavior of Ceramic Matrix Composites

Nik Chawla Department of Material Science and Engineering, Arizona State University, Tempe, AZ, USA

Thermal Shock Behavior of Ceramic Matrix Composites

Vasyl F. Chekurin Pidstryhach Institute for Applied Problems of Mechanics and Mathematics, National Academy of Sciences of Ukraine, Lviv, Ukraine

Thermoelasticity of Semiconductors: The Many-Continuum Thermodynamic Approach

Fu-Mo Chen Department of Mechanical Engineering, Nan-Kai University of Technology, Tsao Tun, Taiwan, Republic of China Heat Conduction Problems with Multiple Inclusions Point Heat Source Interacting with a Nonuniformly Coated Circular Inclusion

Jinn-Kuen Chen Department of Mechanical and Aerospace Engineering, University of Missouri, Columbia, MO, USA

Atomic-Level Hybrid Modeling of Thermomechanical Stress Wave in Metal Thin Films Induced by Ultrashort Laser Pulses

Peter Y. P. Chen School of Electrical Engineering \& Telecommunications, University of New South Wales, Sydney, Australia Axisymmetric Thermal Stresses in an Anisotropic Finite Hollow Cylinder 
Wei Q. Chen Department of Engineering Mechanics, Zhejiang University, Hangzhou, Zhejiang, People's Republic of China

General Solution of 3D Thermoelasticity

Xiaohong Chen UTC Aerospace Systems, Chula Vista, CA, USA

Path-Independent Integral

O. P. Chervinko Timoshenko Institute of Mechanics, National Academy of Sciences of Ukraine, Kiev, Ukraine

Modeling of Thermomechanical Process in Growing Viscoplastic Bodies with Accounting of Microstructural Transformation

Kuo-Ning Chiang Advanced Microsystem Packaging and Nano-Mechanics Research Lab, Department of Power Mechanical Engineering, Advanced Packaging Research Center, National Tsing Hua University, Hsin Chu, Taiwan, Republic of China

Thermal Stress-Induced Interfacial Failure Modes of Advanced Electronic Devices

Ryoichi Chiba Department of Mechanical Systems Engineering, Asahikawa National College of Technology, Asahikawa, Hokkaido, Japan Stochastic Analysis of Thermal Stresses in Bodies

Stan Chiriţă Department of Mathematics, Alexandru Ioan Cuza University of Iaşi, Iaşi, Romania

Octav Mayer Institute of Mathematics, Romanian Academy, Iaşi, Romania

Alternative Formulations: Reciprocal Relations

Well-Posed Problems

Michele Chiumenti ETS Ingenieros de Caminos, Canales y Puertos, Universidad Politécnica de Cataluña, Barcelona Tech, Barcelona, Spain

International Center for Numerical Methods in Engineering (CIMNE), Barcelona, Spain

Shaped Metal Deposition Processes

Hanbum Cho King \& Spalding LLP, Redwood City, CA, USA

Hertzian Contact with Heat Flow

Ivan C. Christov Department of Mechanical and Aerospace Engineering, Princeton University, Princeton, NJ, USA

Wave Solutions

Michał Ciałkowski Poznań University of Technology, Poznań, Poland Boundary Element Method in Inverse Heat Conduction Problem Green's Functions in Steady-State Heat Conduction

Michele Ciavarella DMMM Politecnico di Bari, Bari, Italy

Thermoelastic Dynamic Instability (TEDI)

Maria Cinefra Department of Mechanical and Aerospace Engineering, Politecnico di Torino, Torino, Italy

Thermomechanical Bending in Functionally Graded Material Shells 
Simona De Cicco Dipartimento di Strutture per l' Ingegneria e l' Architettura, Università degli Studi di Napoli "Federico II", Napoli, Italy

Problem of Stress Concentration in Elastostatics of Bodies with Microstructure

Singular Surfaces in Thermoviscoelastic Materials with Voids

Variational Principles in the Theory of Elastic Bodies with Microstructure

Vito Antonio Cimmelli Department of Mathematics, Computer Science and Economics, University of Basilicata, Potenza, Italy

Nonequilibrium Temperatures and Second-Sound Propagation Along Nanowires

David L. Clements School of Mathematics, The University of Adelaide, Adelaide, SA, Australia

Anisotropic Thermoelastic Contact Problems

Can Ozgur Colpan Department of Mechanical Engineering, Dokuz Eylul University, Izmir, Turkey

Probability of Failure During the Operation of Direct Internal Reforming Solid Oxide Fuel Cells

Carlos Correa Centro Láser UPM, Madrid, Spain

Induction of Thermo-Mechanical Residual Stresses in Metallic Materials by Laser Shock Processing

Carmela Currò Department of Mathematics and Informatics, University of Messina, Messina, Italy

Acceleration Waves in Layers of Isotropic Solids at Finite Temperatures

Julio Cesar Ramalho Cyrino Ocean Engineering Program, Federal University of Rio de Janeiro, Rio de Janeiro, RJ, Brazil

Thermal Buckling of Beams on Elastic Foundation

Constantine M. Dafermos Division of Applied Mathematics, Brown University, Providence, RI, USA

Regularity of Solutions to the Equations of Thermoelasticity

Weizhong Dai College of Engineering and Science, Louisiana Tech University, Ruston, LA, USA

Finite Difference Simulation of Thermal Deformation in Ultrashort-Pulsed Laser Material Processing

Skin Burn Injury Prediction

Alexandre Danescu Lyon Institute of Nanotechnology, Ecully, France Generalized Thermoelastodynamic Stefan Models

Feras H. Darwish Jordan University of Science and Technology, Irbid, Jordan Thermal Stress Under the Effect of Nonconventional Heat Conduction Models

Balasubramanian Datchanamourty Champaign Simulation Center, Champaign, IL, USA

Piezothermoelastic Analysis: Plate Theories 
Marcos Díaz Centro Láser, UPM, Madrid, Spain

Induction of Thermo-Mechanical Residual Stresses in Metallic Materials by Laser Shock Processing

H. Dehbani Postgraduate School, South Tehran Branch, Islamic Azad University, Tehran, Iran

Exact Solution for Classic Coupled Thermoelasticity in Spherical Coordinates

Exact Solution for Classic Coupled Thermoporoelasticity in Axisymmetric Cylinder

Exact Solution for Classic Coupled Thermoporoelasticity in Cylindrical Coordinates

Exact Solution for Lord-Shulman Generalized Coupled Thermoporoelasticity in Cylindrical Coordinates

Exact Solution for Lord-Shulman Generalized Coupled Thermoporoelasticity in Spherical Coordinates

Exact Solution for Quasi-Static Porothermoelasticity in Spherical Coordinates

Antoine Deheeger Clermont Université, Université Blaise Pascal, CNRS, Institut Pascal, Clermont-Ferrand, France

Thermal Stresses in Hybrid Composite Joints

Krzysztof Dems Department of Technical Mechanics and Informatics, Technical University of Łódź, Łódź, Poland

Sensitivity Analysis for Thermoelastic State Fields

Sensitivity of Thermal Response with Respect to Material Parameters and Shape of Interfaces and Boundaries

Thermal Properties of Homogenized Model of Fiber Reinforced Composite Material

Dean Deng College of Materials Science and Engineering, Chongqing University, Chongqing, People's Republic of China

Welding of Dissimilar Metals and Post Weld Heat Treatment

I. I. Derjavko Scientific Institute of Scientific Industrial Association "LUCH", Podolsk, Russia

National Nuclear Center of the Republic of Kazakhstan, Kurchatov, Kazakhstan

Influence of Residual Stresses on Fracture at Thermal Loading Modeling of Residual Stress Formation on Thermal-Stress Resistance of Nuclear Fuel Elements

Alexis Deschamps SIMAP, INP Grenoble - CNRS - UJF, Grenoble, France Microstructural Evolution in Heat-Treatable Aluminum Alloys

Aproop Dheeraj Indian Institute of Technology, Kharagpur, India Thermomechanical Inelastic Buckling of Beams Resting on an Elastic Foundation 
Ibrahim Dincer University of Ontario, Institute of Technology, Oshawa, Ontario, Canada

Probability of Failure During the Operation of Direct Internal Reforming Solid Oxide Fuel Cells

Haojiang Ding Department of Civil Engineering, Zhejiang University, Hangzhou, People's Republic of China

Piezothermoelastic Problems: Dynamic Analyses

M. Cengiz Dökmeci Istanbul Technical University, Istanbul, Turkey

Piezothermoelasticity with Hygro-Effects: Fundamental Theory

Piezothermoelasticity: Fundamental Theory

Piezothermoelasticity: Variational Principles

Variational Principles in Coupled Thermoelasticity

Chunying Dong Department of Mechanics, School of Aerospace Engineering, Beijing Institute of Technology, Beijing, People's Republic of China Cracks in Transversely Isotropic and Inhomogeneous Elastic Solids

Pingsha Dong College of Engineering, University of New Orleans, New Orleans, LA, USA

Residual in Weld Repairs

Michele D'Ottavio Laboratoire Energétique Mécanique Electromagnétisme (LEME) - EA4416, Université Paris Ouest - Nanterre - La Défense, Ville d'Avray, France

Thermal Stress Analysis of Homogeneous and Laminated Shells by Finite Element Method

James F. Doyle Purdue University, West Lafayette, IN, USA Viscoelastic Wave Propagation

Bogdan Drobenko Pidstryhach Institute for Applied Problems of Mechanics and Mathematics, National Academy of Sciences of Ukraine, Lviv, Ukraine

Thermomechanics of Electroconductive Solids

J. Dubois INSA-Lyon MATEIS CNRS UMR5510, Villeurbanne, France Thermal Shock and Thermal Fatigue Study of Ceramic Materials on a Newly Developed Ascending Thermal Shock Test Equipment

Piotr Duda Institute of Thermal Power Engineering, Faculty of Mechanical Engineering, Cracow University of Technology, Cracow, Poland

Inverse Determination of Temperature and Thermal Stress Distributions in

Solids with Simple and Complex Geometries

Overdetermined Inverse Conduction Problems

Space- and Time-Marching Methods for One-Dimensional Inverse Heat

Conduction Problems

P. Dufrénoy Laboratoire de Mécanique de Lille, Université de Lille Nord, Lille, France

Experimental Analysis of Hot Spotting in Sliding Systems 
Janusz Dyszlewicz Faculty of Fundamental Problems of Technology, Institute of Mathematics and Computer Science, Wrocław Technical University, Wrocław, Poland

Coupled Dynamic Micropolar Problems of Thermoelasticity:

Stress-Temperature Equations of Motion of Ignaczak Type

Dynamic Micropolar Thermoelasticity

Selected Problems of Elastostatics and Thermoelastostatics of the

Hemitropic Micropolar Medium

Piotr Dzierwa Institute of Process and Power Engineering, Cracow University of Technology, Cracow, Poland

Optimum Heating of Pressure Components of Complex Shape

Quasi-Steady-State Approach for Solving Transient Heat Conduction

Problems

Halina Egner Institute of Applied Mechanics, Cracow University of Technology, Cracow, Poland

Internal State Variables and Thermodynamic Forces

Non-Isothermal Coupled Thermo-Damage-Plasticity

V. S. Egorov Scientific Institute of Scientific Industrial Association "LUCH", Podolsk, Russia

Fracture of Elastic-Brittle Bodies at Combined Thermal and Mechanical Loadings

Growing Cracks and Redistribution of Thermal Stresses in Thermal-Loaded Disk

Mohammed A. Elhagary Department of Mathematics, Faculty of Science, Damietta University, New Damietta, Egypt Asymptotic Expansions in Coupled and Generalized Thermoelasticity Generalized Theory of Thermoelasticity with One Relaxation Time

Ahmed S. El-Karamany Department of Mathematical and Physical Sciences, Nizwa University, Nizwa, Oman

Coupled and Generalized Thermoviscoelasticity

Tov Elperin Department of Mechanical Engineering, Pearlstone Center for Aeronautical Engineering Studies, Ben-Gurion University of the Negev, Beer-Sheva, Israel

Thermal Stress Caused by Laser Pulse in Functionally Graded Materials

Ibrahim H. El-sirafy Department of Mathematics, Alexandria University, Alexandria, Egypt

Goursat Functions of Thermoelastic Problem of an Infinite Plate with Hypitrochoidal Hole

Igor Emri Center for Experimental Mechanics, Faculty of Mechanical Engineering, University of Ljubljana, Ljubljana, Slovenia Experimental Determination of Material Time-Dependent Properties 
Göran Engberg Department of Material Science, Dalarna University, Falun, Sweden

Constitutive Models, Physically Based Models for Plasticity

Victor A. Eremeyev Otto-von-Guericke University Magdeburg, Magdeburg, Germany

South Scientific Center of RASci and South Federal University, Rostov on Don, Russia

Acceleration Waves in Nonlinear Thermoelastic Micropolar Media

Ellipticity Condition and Acceleration Waves in Nonlinear Thermoelastic

Solids

Phase Transitions in Thermoviscoelastic Shells

Andrei Errapart Institute of Cybernetics, Tallinn University of Technology, Tallinn, Estonia

Photoelasticity for the Measurement of Thermal Residual Stresses in Glass

Mohammad Reza Eslami Department of Mechanical Engineering, Amirkabir University of Technology, Tehran, Iran

Circular/Annular Plates, Thermal Buckling

Effect of Creep on Cyclic Loading of Spherical Vessels Based on the

Kinematic Hardening Models

Effect of Creep on Thermal Cyclic Loading of Beams Based on the

Kinematic Hardening Models

Effect of Creep on Thermal Cyclic Loading of Rotating Disks

Effect of Creep on Thermal Cyclic Loading of Thick Cylindrical Vessels

Based on the Kinematic Hardening Models

Effects of Elastic Foundation on Thermal Buckling of Circular/Annular Plates

Effects of Ring Supports on Thermo-Elastic Buckling of Circular Plate

Generalized Thermoelasticity of a Crack Problem Considering

Lord-Shulman Theory

Higher-Order Beam Theories

Linear Thermal Buckling of Truncated FGM Conical Shells

Linear Thermal Buckling of Truncated Isotropic Conical Shells with

Piezoelectric Layers

Numerical Simulation of Cyclic Loading of Thermal Stresses

Pyroelectric Effect on Dynamic Response of Coupled Distributed

Composite Plate

Rings, Thermal Buckling

Slender Beams, Thermal Buckling

Thermal Buckling and Dynamic Post-Buckling Analysis of Piezoelectric FGM Hybrid Cylindrical Shells

Thermal Buckling of Perfect and Imperfect Isotropic Shallow Spherical Shells

Thermal Cyclic Loading of Beams Based on the Chaboche Kinematic Hardening Model

Thermal Cyclic Loading of Beams Based on the Prager and ArmstrongFrederick Kinematic Hardening Models 
Thermal Cyclic Loading of Rotating Disks

Thermal Cyclic Loading of Thick Cylindrical Vessels Based on the Prager and Armstrong-Frederick Kinematic Hardening Models

Thermal Cyclic Loading of Thick Spherical Vessels Based on the Prager and Armstrong-Frederick Kinematic Hardening Models

Thermal Effects on Buckling of Cylindrical Shells Surrounded by Pasternak Medium

Thermal Fracture by Extended FEM

Thermal Stresses: First 125 Years of Research (from 1837 to Approximately 1962)

Thermoelastic Buckling of Imperfect FGM Cylindrical Shells

Thermoelastic Stability Analysis of Perfect Isotropic Deep Spherical Shell Thermoelectrical Buckling and Postbuckling of F unctionally Graded Piezoelectric Beams

Thermo-electro-mechanical Buckling of Circular Plates

Thin Cylindrical Shells, Thermal Buckling

Magdy A. Ezzat Department of Mathematics, Faculty of Education, Alexandria University, Alexandria, Egypt

Department of Mathematics, Faculty of Science and Letter in Al Bukayriyyah, Al-Qassim University, Al-Qassim, Saudi Arabia

Electromagneto Coupled and Generalized Thermoelasticity

Xuejun Fan Department of Mechanical Engineering, Lamar University, Beaumont, TX, USA

Thermal Stresses in Flip Chip BGA Packaging

Thermal Stresses in Wafer-Level Packaging

CuiYing Fan Henan Key Engineering Laboratory for Anti-fatigue Manufacturing Technology and the School of Mechanical Engineering, Zhengzhou University, Zhengzhou, People's Republic of China

Extended Displacement Discontinuity Boundary Integral Equation Method for Analysis of Cracks in Smart Materials

Nonlinear Fracture Models of Magnetoelectroelastic Media

Gilbert Fantozzi INSA-Lyon MATEIS CNRS UMR5510, Villeurbanne, France

Thermal Shock and Thermal Fatigue Study of Ceramic Materials on a Newly Developed Ascending Thermal Shock Test Equipment

Seyyed Ahmad Fazelzadeh Department of Mechanical Engineering, Shiraz University, Shiraz, Iran Aerothermoelastic Behaviors of Functionally Graded Panel Structures Fluid-Thermal Structural Coupling in the Modeling of Carbon Nanotubes Fluid-Thermo-Elastic and Aero-Thermo-Elastic Governing Equations for FGM Structures

Fluid-Thermoelastic Behaviors of FGM Thin-Walled Beams and Pipes Functionally Graded Structures: Aerothermoelastic Interactions 
Cristian Făciu "Simion Stoilow" Institute of Mathematics of the Romanian Academy, Research Unit No. 6, Bucharest, Romania

Heat Conduction and Viscosity as Structuring Mechanisms for Shock Waves in Thermoelastic Materials

Maxwellian Rate-Type Thermo-Viscoelastic Bar Theory: An Approach to Non-monotone Thermoelasticity

Pseudoelasticity and Shape Memory Effect: A Maxwellian Rate-Type Approach

Thermoelastic Bar Theory

Fausto Ferrari Dipartimento di Matematica dell'Università di Bologna Piazza di Porta S. Donato, Bologna, Italy

Fourier Transform

Eric Feulvarch Université de Lyon, ENISE, LTDS, UMR 5513 CNRS, Saint-Etienne, France

Modeling and Numerical Simulation of Resistance Spot Welding Process

John F. Field SMF Fracture and Shock Physics Group, Cavendish Laboratory, University of Cambridge, Cambridge, UK

Hot Spot Ignition of Explosives and Their Thermal Properties and Experimental Measurement of the Thermal Properties of a PBX and Its Binder System

Martin Fisk School of Technology, Malmö University, Malmö, Sweden Induction Heating

Repair Welding and Local Heat Treatment

Volodymyr Flyachok Department of Applied Mathematics, Ukrainian Academy of Printing, Lviv, Ukraine

Thermoelasticity of Thin Shells

Samuel Forest Mines ParisTech/CNRS, Centre des Matériaux/CNRS UMR 7633, Evry Cedex, France

Asymptotic Analysis of Heterogeneous Micromorphic Elastic Solids Gradient Thermoplasticity

Andrzej Frąckowiak Poznań University of Technology, Poznań, Poland Boundary Element Method in Inverse Heat Conduction Problem Green's Functions in Steady-State Heat Conduction

Yehoshua Frostig Faculty of Civil and Environmental Engineering, Technion - Israel Institute of Technology, Haifa, Israel

Thermomechanical Nonlinear Response of Sandwich Panels

Piotr Furmański Institute of Heat Engineering, Faculty of Power and Aeronautical Engineering, Warsaw University of Technology, Warsaw, Poland Heterogeneous Media and Their Thermal Properties Thermal Contact Resistance

Toshio Furukawa Department of Mechanical Systems Engineering, University of the Ryukyus, Nishihara, Okinawa, Japan

Body Force Analogy for Thermoelasticity 
Cătălin Galeş Department of Mathematics, Faculty of Mathematics, “Al. I. Cuza” University of Iaşi, Iaşi, Romania

Continuous Dependence Results

Hamilton-Kirchhoff Principle

Nonlinear Thermoelastic Model

Structural Stability in Linear Thermoelasticity

Uniqueness and Continuous Dependence Results in Nonlinear

Thermoviscoelasticity

Laurent Gallais Institut Fresnel (CNRS, Ecole Centrale Marseille, Université Aix-Marseille), Ecole Centrale Marseille, Campus Universitaire de Saint Jérôme, Marseille, France

Stress Induced by $\mathrm{CO}_{2}$ Laser Silica Processing

Luigi Gambarotta Department of Civil, Chemical and Environmental Engineering, University of Genoa, Genoa, Italy

Thermo-Inelasticity and Damage

Zhenghao Gan Technology R\&D Center, Semiconductor Manufacturing International Corp., Shanghai, People's Republic of China

Inelastic Analysis of Functionally Graded Thermal Barrier Coating (FG TBC) Under Thermal Shock

Yong Gan School of Aeronautics and Astronautics, Zhejiang University, Hangzhou, Zhejiang, People's Republic of China

Atomic-Level Hybrid Modeling of Thermomechanical Stress Wave in Metal Thin Films Induced by Ultrashort Laser Pulses

Artur Ganczarski Institute of Applied Mechanics, Cracow University of Technology, Cracow, Poland

Anisotropic Initial Yield and Failure Criteria Including Temperature Effect

Dariusz Gawin Department of Building Physics and Building Materials, Technical University of Łódź, Łódź, Poland

Multiphase Porous Media, High Temperature

Stelios K. Georgantzinos Department of Mechanical Engineering and Aeronautics, University of Patras, Patras, Greece

Crack Closure

Haralambos Georgiadis Mechanics Division, National Technical University, Athens, Greece

Rapid Sliding Contact: Elastodynamic Steady State

Marina Gergesova Center for Experimental Mechanics, Faculty of Mechanical Engineering, University of Ljubljana, Ljubljana, Slovenia

Experimental Determination of Material Time-Dependent Properties

P. Ghaedi Department of Mechanical Engineering, I.A.U, Tehran South Branch, Tehran, Iran

Thermal Buckling of Perfect and Imperfect Isotropic Shallow Spherical Shells 
Reza Ghaffarian Jet Propulsion Laboratory, California Institute of Technology, Pasadena, CA, USA

Area Array Package/Assembly Under Thermal Stress

Column Grid Array Assembly Under Thermal Cycling Stress

A. F. Ghaleb Department of Mathematics, Faculty of Science, Cairo University, Cairo, Giza, Egypt

Boundary Integral Formulation of the Plane Problems of Thermoelastostatics

Coupled Thermoelectroelasticity in Extended Thermodynamics

Esmaeal Ghavanloo Department of Mechanical Engineering, Shiraz University, Shiraz, Iran

Fluid-Thermal Structural Coupling in the Modeling of Carbon Nanotubes

Ionel-Dumitrel Ghiba Department of Mathematics, "Alexandru Ioan Cuza" University of Iaşi, Iaşi, Romania

"Octav Mayer" Institute of Mathematics, Romanian Academy, Iaşi, Romania Boundary-Initial Value Problems of Thermoelastodynamics

Linear Thermoelastic Model

Partition of Energy

Saint-Venant's Principle

Thermoelastic Waves

Andrea Gil-Santos Centro Láser UPM, Madrid, Spain

Induction of Thermo-Mechanical Residual Stresses in Metallic Materials by Laser Shock Processing

Claudio Giorgi Università degli Studi di Brescia, Brescia, Italy Mathematical Models of Reissner-Mindlin Thermoviscoelastic Plates Ordinary Differential Equations (ODE)

Victor Giurgiutiu Department of Mechanical Engineering, College of Engineering and Computing, University of South Carolina, Columbia, SC, USA

Piezoelectric Materials: Effect of Temperature

Tekin Gültop Department of Civil Engineering, Gazi University, Ankara, Turkey

Propagation of Shock Waves in Thermoelastic Solids in View of Singular Surfaces

Zbigniew Gnutek Institute of Heat Engineering and Fluid Mechanics, Wroclaw University of Technology, Wrocław, Poland

Temperature

Thermal Science

Thermodynamic System

George A. Gogotsi Pisarenko Institute for Problems of Strength, National Academy of Sciences of Ukraine, Kiev, Ukraine

Brittleness Measure of Ceramics 
John Goldak Goldak Technologies Inc., Ottawa, Ontario, Canada

Computational Welding Mechanics

Joamin Gonzalez-Gutierrez Center for Experimental Mechanics, Faculty of Mechanical Engineering, University of Ljubljana, Ljubljana, Slovenia Experimental Determination of Material Time-Dependent Properties

Michel Grédiac Clermont Université, Université Blaise Pascal, CNRS, Institut Pascal, Clermont-Ferrand, France

Thermal Stresses in Hybrid Composite Joints

Sławomir Grądziel Institute of Thermal Power Engineering, Faculty of Mechanical Engineering, Cracow University of Technology, Cracow, Poland Energetics

Multidimensional Heat Conduction Problems with Boundary Conditions of the Third Kind

J. A. Greenwood Department of Engineering, University of Cambridge, Cambridge, UK

Surface Temperatures in Sliding

Alexander Grigorenko Timoshenko Institute of Mechanics, National Academy of Sciences of Ukraine, Kiev, Ukraine

Numerical Approaches to Solving Thermostress Problems for

Inhomogeneous Anisotropic Shells Using Various Models

Yaroslav Grigorenko Timoshenko Institute of Mechanics, National Academy of Sciences of Ukraine, Kiev, Ukraine

Numerical Approaches to Solving Thermostress Problems for Inhomogeneous Anisotropic Shells Using Various Models

Viktor T. Grinchenko Institute of Hydromechanics, National Academy of Sciences of Ukraine, Kiev, Ukraine

Finite Elastic Solids, Thermal Stress

Janez Grum University of Ljubljana, Ljubljana, Slovenia Induction Surface Hardening

Krzysztof Grysa Kielce University of Technology, Kielce, Poland Trefftz Method in Solving Inverse Heat Conduction Problems

Piotr Grzes Faculty of Mechanical Engineering, Bialystok University of Technology, Białystok, Poland

FEM-Modeling of Frictional Heating During Braking

Davide Guidetti Dipartimento di Matematica, Università di Bologna, Bologna, Italy

Distributions

Linear Partial Differential Equations: Fundamental Solutions, Hypoellipticity, Local Existence

Operations on Distributions 
Jagadish Babu Gunda Advanced Systems Laboratory, Kanchanbagh, Hyderabad, India

Thermal Post-Buckling Paths of Beams

Thermal Post-Buckling Paths of Square Plates

Licheng Guo Department of Astronautic Science and Mechanics, Harbin Institute of Technology, Harbin, People's Republic of China

FGM Plate with Surface Crack, Thermal Shock

Multilayered Method for FGM

Yuebin B. Guo Department of Mechanical Engineering, The University of Alabama, Tuscaloosa, AL, USA

Laser Shock Peening

Sachin Gupta Dynamics Photomechanics Laboratory, Department of Mechanical, Industrial and Systems Engineering, University of Rhode Island, Kingston, RI, USA

Performance of Sandwich Structures Under Dynamic Loads at Different Temperatures

Igor A. Guz University of Aberdeen, Scotland, UK

Dissipative Heating of Thin-Wall Structures Containing Piezoactive Layers

Oleksandr Hachkevych Pidstryhach Institute for Applied Problems of Mechanics and Mathematics, National Academy of Sciences of Ukraine, Lviv, Ukraine

Thermomechanics of Electroconductive Solids

Feridun Hamdullahpur Mechanical and Mechatronics Engineering Department, University of Waterloo, Waterloo, Ontario, Canada

Probability of Failure During the Operation of Direct Internal Reforming Solid Oxide Fuel Cells

Farid A. Hamza Department of Mathematics, Faculty of Science, University of Alexandria, Alexandria, Egypt

Axisymmetric Generalized Thermoelasticity Problems in Spherical Regions Axisymmetric Generalized Thermoelasticity Problems Using Cylindrical

Coordinates

Zhili Hao Department of Mechanical and Aerospace Engineering, Old Dominion University, Norfolk, VA, USA

Electro-Thermo-Mechanical System: Thermoelastic Damping in

Resonators

Davresh Hasanyan Department of Material Science and Engineering, Virginia Polytechnic Institute and State University, Blacksburg, VA, USA Electroconductive Composites Subjected to Magnetoelastic and Thermomechanical Loadings: Nonlinear Response and Stability 
Magneto-thermo-elastic Equations of Thin Electroconductive Isotropic

Shells and Plates: Linear Equations

Magneto-Thermoelastic Equations of Thin Electroconductive Isotropic

Shells and Plates: Nonlinear Equations

Magneto-Thermo-Elasticity of Laminated Composite Plates Incorporating Structural Nonlinearity

Thermo-Magneto-Electro-Elastic Multilayer Composites: Effective Properties and Magneto-electric Coefficients

Norio Hasebe Department of Civil Engineering, Nagoya Institute of Technology, Showa-ku, Nagoya, Japan

External Force and Displacement Boundary Value Problems

Green's Function of Heat Source for Mixed Boundary Value Problem

Green's Function of Thermoelastic Mixed Boundary Value Problem for Elliptic Hole

Green's Function for Thermal and Mechanical Mixed Boundary Value

Problem for an Elliptic Hole

Heat Conduction Problem

Heat Conduction Problem for a Strip with a Notch

Interaction Between a Rigid Inclusion and a Line Crack Under Uniform

Heat Flux

Interaction Problem Between a Hole and a Crack

Solution of an Elliptical Rigid Inclusion with Debondings

Thermal Stress for Mixed Heat Conduction Boundary Around an

Arbitrarily Shaped Hole Under Uniform Heat Flux

Thermal Stresses Under Electric Current

Noha M. Hassan Faculty of Engineering and Applied Sciences, ALHOSN University, Abu Dhabi, United Arab Emirates

Porous Thermoelasticity with Applications

Toshiaki Hata Department of Engineering Education, Faculty of Education, Shizuoka University, Shizuoka City, Japan

One-Dimensional Thermal Stresses in Spheres

Thermal Stress-Focusing Effect

Jesper H. Hattel Department of Mechanical Engineering, Section of Manufacturing Engineering, Technical University of Denmark, Lyngby, Denmark

Modeling Residual Stresses in Friction Stir Welding of Al Alloys

Louis G. Hector Jr. General Motors R\&D Center, Warren, MI, USA

Thermoelastic Stresses in a Bonded Layer Due to Repetitively Pulsed Laser Radiation

Thermomechanical Growth Instability in Solidification

Amin Heidarpour Department of Civil Engineering, Monash University, Melbourne, VIC, Australia

Local Thermal Stability of Flanges and Webs

Thermal Flexural-Torsional Stability of Arches 
Fariborz Heidary Mechanical Engineering Department, Niayesh Engineering Campus, Central Branch of Azad University, Tehran, Iran Pyroelectric Effect on Dynamic Response of Coupled Distributed Composite Plate

Klaus P. Herrmann Lehrstuhl fuer Technische Mechanik, Paderborn University, Paderborn, Germany

Contact Zone Model for an Interface Crack in a Piezoelectric Bimaterial Under Thermoelectromechanical Loadings

Richard B. Hetnarski Department of Mechanical Engineering, Rochester Institute of Technology, Rochester, NY, USA

Naples, FL, USA

Coupled Problem of Thermoelasticity: Solution in a Series of Functions Form

Direct Approach to Thermoelasticity

Generalized Thermoelasticity of a Crack Problem Considering LordShulman Theory

Generalized Thermoelasticity: Mathematical Formulation

Laplace Transforms of Specific Exponential Form Encountered in Thermoelasticity

State-Space Approach to Thermoelasticity

Thermal Stresses: First 125 Years of Research (from 1837 to Approximately 1962)

Thermoelastic Stresses in a Bonded Layer Due to Repetitively Pulsed Laser Radiation

Harry H. Hilton Aerospace Engineering Department, College of Engineering and Private Sector Program Division, National Center for Supercomputing Applications (NCSA), University of Illinois at UrbanaChampaign (UIUC), Urbana, IL, USA

Aeroviscoelasticity Designer FGMs: Passive Control Through Tailored Functionally Graded Materials

Linear Aero-Thermo-Servo-Viscoelasticity, Part I: General Theory

Linear Aero-Thermo-Servo-Viscoelasticity, Part II: Dynamic Considerations: Lifting Surface and Panel Flutter and Aerodynamic Noise

Paul Håkansson Division of Solid Mechanics, Lund University, Lund, Sweden

Isotropic/Kinematic Hardening in Thermoplasticity

Paul S. Ho Microelectronics Research Center, University of Texas, Austin, TX, USA

Thermal Stress in 3-D Packaging

Parisa Hosseini Tehrani School of Railway Engineering, Iran University of Science and Technology, Tehran, Iran Dynamic Fracture Mechanics 
Mohammad Hosseini Department of Mechanical Engineering, Sirjan University of Technology, Sirjan, Iran

Aerothermoelastic Behaviors of Functionally Graded Panel Structures

Fluid-Thermo-Elastic and Aero-Thermo-Elastic Governing Equations for FGM Structures

Fluid-Thermoelastic Behaviors of FGM Thin-Walled Beams and Pipes

Functionally Graded Structures: Aerothermoelastic Interactions

Seyed Mahmoud Hosseini Industrial Engineering Department, Ferdowsi University of Mashhad, Mashhad, Iran

Application of Boundary Integral Equation (BIE) Method in

Thermoelastodynamic Problem

Application of Meshless Local Petrov-Galerkin (MLPG) and Generalized

Finite Difference (GFD) Methods in Coupled Thermoelasticity Analysis of Thick Hollow Cylinder

Deterministic and Stochastic Coupled Thermoelasticity Analysis in Thick

Hollow Cylinder Subjected to Thermal Shock Loading Using Green-

Naghdi Theory

Thermoelastic Wave Propagation Analysis in Thick Hollow Cylinder Based on Green-Naghdi Theory of Coupled Thermoelasticity Using Analytical Method

Peng-Fei Hou Department of Engineering Mechanics, Hunan University, Changsha, Hunan, People's Republic of China

Magneto-electro-thermoelastic Problems: Fundamental Solutions and Green's Function

Thermoelastostatics of Transversely Isotropic Materials: F undamental Solutions and Green's Functions

Meftah Hrairi Department of Mechanical Engineering, International Islamic University Malaysia, Kuala Lumpur, Malaysia

Edge Crack, Bimaterial Systems

Edge Crack, Composite Materials

Edge Crack, Isotropic Material

Shih-Ming Hsu Department of Mechanical Engineering, National Chiao Tung University, Hsinchu, Taiwan, Republic of China

Thermoelastic Waves in Thin Bodies

Chun-Hway Hsueh Department of Materials Science and Engineering, National Taiwan University, Taipei, Taiwan, Republic of China

Delamination at Free Edges

Rui Huang Department of Aerospace Engineering and Engineering Mechanics, University of Texas, Austin, TX, USA

Thermal Stress in 3-D Packaging

Nan-Nong Huang Department of Mechanical and Mechatronic Engineering, National Taiwan Ocean University, Keelung, Taiwan, Republic of China Maysel's Method for Plates

Post-Buckling, Laminated Plates

Stability, Heterogeneous Anisotropic Plates 
Chyanbin Hwu Institute of Aeronautics and Astronautics, National Cheng Kung University, Tainan, Taiwan, Republic of China

Stroh Formalism for Thermoelastic Problems

Thomas H. Hyde Department of Mechanical, Materials and Manufacturing Engineering, University of Nottingham, Nottingham, UK

Finite Element Simulation of the Fusion Welding of Metal Components Including Post-weld Heat Treatment

Gianni Luca Iaccarino Applied High-Performance Scientific Computing Research Laboratory, Department of Applied Science, University of Naples "Parthenope", Naples, Italy

Signorini's Method for Live Loads and Second-Order Effects in Elastostatics

Józef Ignaczak Institute of Fundamental Technological Research, IPPT, Polish Academy of Sciences, Warsaw, Poland

Domain of Influence Theorems in Generalized Thermoelasticity

Generalized Thermoelasticity: Mathematical Formulation

Jay Im Microelectronics Research Center, University of Texas, Austin, TX, USA

Thermal Stress in 3-D Packaging

Esin Inan Civil Engineering Department, Isik University, Istanbul, Turkey Eshelby Tensors in Microcontinuum

Identification of Thermo-microstretch Moduli of Materials by the Use of Vibrational Data of Plates

Tatsuo Inoue Saitama Institute of Technology, Fukaya, Saitama, Japan Foundation of Thermoplasticity

Metallo-Thermo-Mechanics

Welding and Casting: Application of Metallo-thermo-mechanics Part I: Fundamental Framework of the Governing Equations and Simulated Results of Welding Process

Welding and Casting: Application of Metallo-thermo-mechanics Part II: Continuous Casting

Welding and Casting: Application of Metallo-thermo-mechanics Part III: Centrifugal Casting Process

Masayuki Ishihara Osaka Prefecture University, Naka-ku, Sakai, Japan Eigenstrains in Thermal Stresses

One-Dimensional Thermal Stresses in Cylinders

Shouetsu Itou Department of Mechanical Engineering, Kanagawa University, Rokkakubashi, Kanagawa-ku, Yokohama, Japan

Plane Strain and Plane Stress Problems in Thermoelasticity

Tsolo P. Ivanov Sofia University, Sofia, Bulgaria Surface Wave Propagation in a Thermoelastic Half-Space 
Mohsen Jabbari Faculty of Engineering, Postgraduate School, South Tehran Branch, Islamic Azad University, Tehran, Iran

Analytical Solution for Two-Dimensional Magneto-thermomechanical

Response in FG Hollow Sphere

Exact Solution for Classic Coupled Thermoelasticity in Cylindrical Coordinates

Exact Solution for Classic Coupled Thermoelasticity in Spherical Coordinates

Exact Solution for Classic Coupled Thermoporoelasticity in Axisymmetric Cylinder

Exact Solution for Classic Coupled Thermoporoelasticity in Cylindrical Coordinates

Exact Solution for Lord-Shulman Generalized Coupled Thermoporoelasticity in Cylindrical Coordinates

Exact Solution for Lord-Shulman Generalized Coupled Thermoporoelasticity in Spherical Coordinates

Exact Solution for Quasi-Static Porothermoelasticity in Spherical Coordinates

Mechanical and Thermal Stresses in a FGPM Hollow Cylinder Due to Radially Symmetric loads

Mechanical and Thermal Stresses in a Functionally Graded Hollow Cylinder Due to Nonaxisymmetric Steady-State Loads

Mechanical and Thermal Stresses in a Functionally Graded Hollow Cylinder Due to Radially Symmetric Loads

Thermal and Mechanical Stresses in a Functionally Graded Sphere

Javad Jahanbakhsh Department of Mechanical Engineering, Tehran South Branch, Islamic Azad University, Tehran, Iran

Thermoelastic Stability Analysis of Perfect Isotropic Deep Spherical Shell

A. Jain Department of Mechanical Engineering, University of Minnesota, Minneapolis, MN, USA

Thermal Contact Applications: Finite Element Formulations

Thermal Contact Applications: Mechanical Contact Models

Thermal Contact Applications: Thermal Contact Models

Pahun Jain Indian Institute of Technology, Kharagpur, India

Thermomechanical Inelastic Buckling of Beams Resting on an Elastic Foundation

Yong Hoon Jang School of Mechanical Engineering, Yonsei University, Seoul, South Korea

Functionally Graded Material Minimizes Thermoelastic Instability

J. Y. Jang Department of Mechanical Engineering, Louisiana State University, Baton Rouge, LA, USA

Thermoelastic Instability in Mechanical Systems with Provision for Surface Roughness 
Magdalena Jaremkiewicz Institute of Thermal Power Engineering, Faculty of Mechanical Engineering, Cracow University of Technology, Cracow, Poland

Measurement of Transient Fluid Temperature

Method of Lines in Heat Conduction

Quan Jiang School of Civil Engineering, College of Science, Nantong University, Nantong, People's Republic of China

Fracture of Electrostrictive Materials

Song Jiang Institute of Applied Physics and Computational Mathematics, Beijing, People's Republic of China

Global Existence and Exponential Stability in Nonlinear Thermoelasticity

Tengfei Jiang Microelectronics Research Center, University of Texas, Austin, TX, USA

Thermal Stress in 3-D Packaging

Zhihe Jin Department of Mechanical Engineering, University of Maine, Orono, ME, USA

Crack-Tip Singular Fields in Functionally Graded Materials

Surface Cracks in a Functionally Graded Plate

Thermal Shock Resistance of Functionally Graded Materials

Amin Joodaky Young Researchers Club, Arak Branch, Islamic Azad University, Arak, Iran

Higher Order Thermal Buckling of Circular Plates

B. Lennart Josefson Material and Computational Mechanics, Chalmers University of Technology, Göteborg, Sweden

Welding of Rails and Effects on Crack Initiation and Propagation

David Jou Departament de Física, Universitat Autònoma de Barcelona, Bellaterra, Catalonia, Spain

Institut d'Estudis Catalans, Barcelona, Catalonia, Spain

Nonequilibrium Temperatures and Second-Sound Propagation Along

Nanowires

Dong-Ying Ju Department of Materials Engineering, Saitama Institute of Technology, Fukaya, Saitama, Japan

Inelastic Simulation Involving in Solid Phase Transformation

Rekha Kahali Von Karman Society, Jalpaiguri, India

Plates on Elastic Foundation

Shells, Elastic Foundation

Thermally Induced Vibration, Laminated Plates

Thermally Induced Vibration, Orthotropic Plates

Stefan Kaloerov Mathematics and Computer Science, Department of Elasticity and Calculus Mathematics, Donetsk National University, Donetsk, Ukraine

Multiply Connected Anisotropic Plates, Thermal Stress 
Bohdan M. Kalynyak Pidstryhach Institute for Applied Problems of Mechanics and Mathematics, National Academy of Sciences of Ukraine, Lviv, Ukraine Nonhomogeneous Solids: Integral Equation Approach

Marcin Kamiński Faculty of Civil Engineering, Architecture and Environmental Engineering, Technical University of Łódź, Łódź, Poland

Generalized Stochastic Perturbation-Based Finite Element Analysis of Structures

T.S. Kannan Materials Science Division, National Aerospace Laboratories, Bangalore, India

Thermal Shock and Thermal Fatigue Study of Ceramic Materials on a Newly Developed Ascending Thermal Shock Test Equipment

Mridula Kanoria Department of Applied Mathematics, University of Calcutta, Kolkata, West Bengal, India

Coupled Generalized Thermoelasticity of Functionally Graded Materials

Tarun Kant Department of Civil Engineering, Indian Institute of Technology Bombay, Mumbai, India

High-Order Theory, Composite Plates

Thick Plates, Reissner-Mindlin Theory, Statical Problems

Santosh Kapuria Department of Applied Mechanics, Indian Institute of Technology Delhi, Huaz Khas, New Delhi, India

Piezothermoelastic Analysis: Discrete Layer Theories

Thermoelectrical Buckling of Beams: Piezoelectric Effects

Michał Karcz Energy Conversion Department, Institute of Fluid Flow Machinery, Polish Academy of Sciences, Gdańsk, Poland

Energy

Magnus Karlberg Engineering Sciences and Mathematics, Luleå University of Technology, Luleå, Sweden

Welding Heat Input Models

Lennart Karlsson Engineering Sciences and Mathematics, Luleå University of Technology, Luleå, Sweden

Computational Welding Mechanics

Vasyl G. Karnaukhov Timoshenko Institute of Mechanics, National Academy of Sciences of Ukraine, Kiev, Ukraine

Forced Harmonic Vibrations and Dissipative Heating of Nonelastic Bodies

Piezothermo-Inelastic Behavior of Structural Elements: Vibrations and

Dissipative Heating

Naoto Kasahara Graduate School of Engineering, Nuclear Engineering and Management, University of Tokyo, Tokyo, Japan

Thermal Plastic Ratcheting

Massoud Kaviany Department of Mechanical Engineering, University of Michigan, Ann Arbor, MI, USA

Heat Transfer During Impact 
Ryuusuke Kawamura Institute of Education and Research for Engineering, University of Miyazaki, Miyazaki, Japan

Analytical Method of FGM

Nonhomogeneous Plate with Temperature-Dependent Properties, Thermal Stress

Akira Kawasaki Department of Materials Processing, Graduate School of Engineering, Tohoku University, Aoba-Ku Sendai, Japan

Thermal Shock and Thermal Cyclic Fracture Behavior of Metal/Ceramic Functionally Graded Materials

Leon M. Keer Northwestern University, Evanston, IL, USA

Steady State Heat Flow and Isolated Crack

Steady State Heat Flow into Concentrated Contact

Ratnesh Khandelwal GE India Technology Centre Pvt. Ltd., Bangalore, India $\mathrm{J}_{\mathrm{k}}$ Integrals for Homogeneous and Bimaterial Bodies

Michael M. Khonsari Department of Mechanical Engineering, Louisiana State University, Baton Rouge, LA, USA

Thermoelastic Instability in Mechanical Systems with Provision for Surface Roughness

Ahmad Reza Khorshidvand Department of Mechanical Engineering, South Tehran Branch, Islamic Azad University, Tehran, Iran

Circular/Annular Plates, Thermal Buckling

Thermo-electro-mechanical Buckling of Circular Plates

Yasser Kiani Department of Mechanical Engineering, Amirkabir University of Technology, Tehran, Iran

Effects of Elastic Foundation on Thermal Buckling of Circular/Annular Plates

Effects of Ring Supports on Thermo-Elastic Buckling of Circular Plate

Higher-Order Beam Theories

Rings, Thermal Buckling

Slender Beams, Thermal Buckling

Thermal Effects on Buckling of Cylindrical Shells Surrounded by Pasternak Medium

Thin Cylindrical Shells, Thermal Buckling

Woo-Seung Kim Department of Mechanical Engineering, Hanyang University, Seoul, South Korea

Thermoelastic Stresses in a Bonded Layer Due to Repetitively Pulsed Laser Radiation

Ahmet Kiris Mathematics Dept, Istanbul Technical University, Maslak, Istanbul, Turkey

Eshelby Tensors in Microcontinuum

J. M. Chandra Kishen Department of Civil Engineering, Indian Institute of Science, Bangalore, India $\mathrm{J}_{\mathrm{k}}$ Integrals for Homogeneous and Bimaterial Bodies 
Hryhoriy Kit Pidstryhach Institute for Applied Problems of Mechanics and Mathematics, National Academy of Sciences of Ukraine, Lviv, Ukraine

Potential Methods in the Spatial Problems of Heat Conduction and Thermoelasticity for Solids with Cracks

Damjan Klobčar Faculty of Mechanical Engineering, University of Ljubljana, Ljubljana, Slovenia

Thermal Fatigue of Materials for Die Casting Tooling

Robin J. Knops The Maxwell Institute of Mathematical Sciences, HeriotWatt University, Edinburgh, Scotland, UK

Continuous Data Dependence in Linear Theories of

Thermoelastodynamics. Part I: Classical Theories. Basics and

Logarithmic Convexity

Continuous Data Dependence in Linear Theories of

Thermoelastodynamics. Part II: Classical Theories, Lagrange Identity

Methods, and Positive-Definite Arguments

Continuous Data Dependence in Linear Theories of

Thermoelastodynamics. Part III: Nonclassical Theories

Spatial and Structural Stability in Thermoelastostatics

Hideo Koguchi Department of Mechanical Engineering, Nagaoka University of Technology, Nagaoka, Niigata, Japan

Singular Stress

Mohammad Komijani Department of Mechanical Engineering, Amirkabir University of Technology, Tehran, Iran

Thermoelectrical Buckling and Postbuckling of Functionally Graded

Piezoelectric Beams

Ming Kong DARPA Center for Integrated Micro/Nano-Electromechanical Transducers (iMINT), Department of Mechanical Engineering, University of Colorado, Boulder, CO, USA

Thermal Stress in MEMS

Piotr Koniorczyk Faculty of Mechatronics and Aerospace, Military University of Technology, Warsaw, Poland

Two-Dimensional, Steady-State Conduction

Two-Dimensional, Steady-State Conduction: Tables

Jan Adam Kołodziej Institute of Applied Mechanics, Poznań University of Technology, Poznań, Poland

Green's Functions for Inverse Heat Conduction Problems

Point, Line, Surface Sources

Wojciech Kosman Institute of Power Engineering and Turbomachinery, Silesian University of Technology, Gliwice, Poland

Transient Temperature and Thermal Stresses in Turbine Components

Shweta Kothari Department of Applied Mathematics, Indian Institute of Technology (Banaras Hindu University), Varanasi, India

Dual Phase-Lag Thermoelasticity 
Katarzyna Kowalczyk-Gajewska Department of Mechanics of Materials, Institute of Fundamental Technological Research, Polish Academy of Sciences, Warsaw, Poland

Thermoplasticity of Polycrystals

Zbigniew L. Kowalewski Department for Strength of Materials, Institute of Fundamental Technological Research, Polish Academy of Sciences, Warsaw, Poland

Thermo-creep Damage in Cu/Al-Alloys

Michal Kuciej Faculty of Mechanical Engineering, Bialystok University of Technology, Białystok, Poland

One-Dimensional Analytical Models of Frictional Heating During Braking

Ismail Kucuk Department of Mathematics and Statistics, American University of Sharjah, Sharjah, United Arab Emirates

Piezoelectric Actuation: Control of Linear Thermal Vibrations

Jakob Kuebler Lab for High Performance Ceramics, EMPA, Duebendorf, Switzerland

Control of Thermal Residual Stresses

Roushan Kumar Department of Mathematics, Central University of Bihar, Patna, India

Dual Phase-Lag Thermoelasticity

Roman Kushnir Pidstryhach Institute for Applied Problems of Mechanics and Mathematics, National Academy of Sciences of Ukraine, Lviv, Ukraine

Application of the Generalized Functions Method for Analysis of Thermal Stresses in Piecewise-Homogeneous Solids

Determination of the Thermal Fields and Stresses in Multilayer Solids by Means of the Constructed Green Functions

Jonathan Lake Los Angeles Electrical Engineering Department, University of California, LA, USA

Theory and Engineering of Micro-scale Resonators with Low

Thermoelastic Dissipation

Anatoly G. Lanin Scientific Institute of Scientific Industrial Association "LUCH", Podolsk, Russia

Fracture of Elastic-Brittle Bodies at Combined Thermal and Mechanical Loadings

Growing Cracks and Redistribution of Thermal Stresses in Thermal-Loaded Disk

Heating Methods of Testing for Thermal Stress Resistance Historic Evolution of Thermal Stress Resistance Concept Influence of Residual Stresses on Fracture at Thermal Loading Modeling of Residual Stress Formation on Thermal-Stress Resistance of Nuclear Fuel Elements 
Barbara Lazzari Department of Mathematics, Alma Mater Studiorum Università di Bologna, Bologna, Italy

Laplace Transform

Chang-Chun Lee Department of Mechanical Engineering, Chung Yuan Christian University, Chungli City, Taoyuan County, Taiwan, Republic of China

Thermal Stress-Induced Interfacial Failure Modes of Advanced Electronic Devices

Jong Seh Lee Department of Civil and Environmental Engineering, Hanyang University, Ansan, South Korea

Magneto-Thermoelastic Instability: Ferromagnetic Plates

Kwangjin Lee Korea Delphi Automotive Systems Corporation, Farmington Hills, MI, USA

Perturbation Methods in TEI, Theoretical Analysis

Yung-Chen Lee DARPA Center for Integrated Micro/Nano-Electromechanical Transducers (iMINT), Department of Mechanical Engineering, University of Colorado, Boulder, CO, USA

Thermal Stress in MEMS

Shuting Lei Department of Industrial and Manufacturing Systems Engineering, Kansas State University, Manhattan, KS, USA

Thermal Stress in Laser-Assisted Machining

Marcin Lemański Energy Conversion Department, Institute of Fluid Flow Machinery, Polish Academy of Sciences, Gdańsk, Poland

Entropy: The Classical View

Cristina Leonelli Dipartimento di Ingegneria "Enzo Ferrari”, Università degli Studi di Modena e Reggio Emilia, Modena, Italy

Microstructural Tailoring of Thermal Shock Resistance in Refractory

Materials

Matteo Leoni Department of Materials Engineering and Industrial Technologies, University of Trento, Trento, TN, Italy

Residual Stress Profile in Ceramic Laminates

Maciej Lewandowski Faculty of Fundamental Problems of Technology, Institute of Mathematics and Computer Science, Wrocław Technical University, Wrocław, Poland

Dynamic Micropolar Thermoelasticity

Chuan Li Department of Biomedical Engineering, National Yang Ming University, Taipei, Taiwan, Republic of China

Department of Mechanical Engineering, National Central University, Jhongli, Taoyuan, Taiwan, Republic of China

Thermoelastic Analysis for Duplex Heat Exchanger Tubes 
Jiefang Li Department of Material Science and Engineering, Virginia Polytechnic Institute and State University, Blacksburg, VA, USA

Electroconductive Composites Subjected to Magnetoelastic and Thermomechanical Loadings: Nonlinear Response and Stability

Magneto-Thermoelastic Equations of Thin Electroconductive Isotropic Shells and Plates: Nonlinear Equations

Thermo-Magneto-Electro-Elastic Multilayer Composites: Effective Properties and Magneto-electric Coefficients

Leijun Li Mechanical and Aerospace Engineering, Utah State University, Logan, UT, USA

Thermomechanical Phenomena in Pulsed Laser Powder Deposition

Wenya Li Shaanxi Key Laboratory of Friction Welding Technologies, School of Materials Science and Engineering, Northwestern Polytechnical University, Xi'an, Shaanxi, People's Republic of China

Linear Friction Welding

X. F. Li School of Civil Engineering, Central South University, Changsha, People's Republic of China

Elastic T-Stresses for a Closed Crack with and Without Kink

Lars-Erik Lindgren Engineering Sciences and Mathematics, Luleå University of Technology, Luleå, Sweden

Modeling of Welding of Austenitic Stainless Steels

Welding and Heat Treatment of Alloys

Welding Stresses

Marian Lipka Institute of Power Engineering and Turbomachinery, Silesian University of Technology, Gliwice, Poland

Design Optimization of Turbine Components with the Use of Temperature and Thermal Stresses Objectives

Thomas Jin-Chee Liu Department of Mechanical Engineering, Ming Chi University of Technology, Taishan, New Taipei City, Taiwan, Republic of China

Crack Detection/Arrest with Joule Heating

Strain Energy Density Factor

Yong Liu Fairchild Semiconductor, South Portland, ME, USA

Thermal Stress Migration and Its Role in Electromigration of Microelectronics

Volodymyr V. Loboda Department of Theoretical and Applied Mechanics, Dniepropetrovsk National University, Dniepropetrovsk, Ukraine

Contact Zone Model for an Interface Crack in a Piezoelectric Bimaterial Under Thermoelectromechanical Loadings

Stanisław Lopata Institute of Thermal Power Engineering, Faculty of Mechanical Engineering, Cracow University of Technology, Cracow, Poland Hyperbolic Heat Conduction Equation 
Vitor Emanuel Lourenço Ocean Engineering Program, Federal University of Rio de Janeiro, Rio de Janeiro, RJ, Brazil

Thermal Buckling of Beams on Elastic Foundation

Yan-Lin Lu College of Mechanical Engineering, Zhejiang University of Technology, Hangzhou, Zhejiang, People's Republic of China

Multiple Virtual Crack Extension Technique

Yong Feng Lu Department of Electrical Engineering, University of Nebraska-Lincoln, Lincoln, NE, USA

Stresses in Laser-Assisted Surface Cleaning

Tanja Lube Institut für Struktur- und Funktionskeramik, Montanuniversitaet Leoben, Leoben, Austria

Effective Fracture Toughness in $\mathrm{Al}_{2} \mathrm{O}_{3}-\mathrm{Al}_{2} \mathrm{O}_{3} / \mathrm{ZrO}_{2}$-Laminates

Szczepan Lubecki Institute of Thermal Power Engineering, Faculty of Mechanical Engineering, Cracow University of Technology, Cracow, Poland Duhamel's Theorem for Time-Dependent Thermal Boundary Conditions

Mykola Lugovy Institute for Problems of Materials Science, Kiev, Ukraine Control of Thermal Residual Stresses

Henryk Lukowicz Institute of Power Engineering and Turbomachinery, Silesian University of Technology, Gliwice, Poland

Transient Temperature and Thermal Stresses in Turbine Components

Roger N. Lumley AWBell Pty Ltd, Dandenong South, VIC, Australia Heat Treatment of Aluminum Alloys

Andreas Lundbäck Department of Engineering Sciences and Mathematics, Mechanics of Solid Materials, Luleå University of Technology, Luleå, Sweden Repair Welding and Local Heat Treatment

Shaped Metal Deposition Processes

Chien-Ching Ma Department of Mechanical Engineering, College of Engineering, National Taiwan University, Taipei, Taiwan, Republic of China Nonhomogeneous Solids: Integral Equation Approach

Artur Maciąg Department of Mathematics, Kielce University of Technology, Kielce, Poland

Trefftz Method in Solving Inverse Heat Conduction Problems

Hossein Mahbadi Mechanical Engineering Department, Islamic Azad University, Central Tehran Branch, Tehran, Iran

Effect of Creep on Cyclic Loading of Spherical Vessels Based on the

Kinematic Hardening Models

Effect of Creep on Thermal Cyclic Loading of Beams Based on the

Kinematic Hardening Models

Effect of Creep on Thermal Cyclic Loading of Rotating Disks

Effect of Creep on Thermal Cyclic Loading of Thick Cylindrical Vessels

Based on the Kinematic Hardening Models

Numerical Simulation of Cyclic Loading of Thermal Stresses 
Thermal Cyclic Loading of Beams Based on the Chaboche Kinematic Hardening Model

Thermal Cyclic Loading of Beams Based on the Prager and Armstrong-Frederick Kinematic Hardening Models

Thermal Cyclic Loading of Rotating Disks

Thermal Cyclic Loading of Thick Cylindrical Vessels Based on the Prager and Armstrong-Frederick Kinematic Hardening Models

Thermal Cyclic Loading of Thick Spherical Vessels Based on the Prager and Armstrong-Frederick Kinematic Hardening Models

Oskar Mahrenholtz Technische Universität Hamburg-Harburg, Meerestechnik II, Germany

Numerical Modelling of Underwater Welding and Cutting

Parviz Malekzadeh Department of Mechanical Engineering, Persian Gulf University, Bushehr, Iran

Thermal Buckling Analysis of Orthotropic Nanoplates on Nonlinear Elastic Foundation

Vladislav Mantič Group of Elasticity and Strength of Materials, School of Engineering, University of Seville, Seville, Spain

Thermal Stresses in Composite Joints

W. G. Mao Key Laboratory of Low Dimensional Materials \& Application Technology, Ministry of Education, Xiangtan University, Hunan, Xiangtan, People's Republic of China

Modeling of Residual Stresses with Thermal Cycling

Carl J. Martin University of Wisconsin - Madison, Madison, WI, USA Thermal Stresses in Solid-Wall Laser IFE Chambers

Bogdan Tadeusz Maruszewski Institute of Applied Mechanics, Poznań University of Technology, Poznań, Poland

Fullerenes: Thermomechanics, Doping, Electrical Conductivity

Piergiovanni Marzocca Department of Mechanical and Aeronautical Engineering, Clarkson University, The Wallace H. Coulter School of Engineering, Potsdam, NY, USA

Aerothermoelastic Behavior of Flat and Curved Panels

Aerothermoelastic Behavior of Lifting Surfaces

Aerothermoelastic Behaviors of Functionally Graded Panel Structures

Aerothermoelastic Control of Lifting Surfaces

Ferromagnetic Plates and Shells

Fluid-Thermal Structural Coupling in the Modeling of Carbon Nanotubes Fluid-Thermo-Elastic and Aero-Thermo-Elastic Governing Equations for FGM Structures

Fluid-Thermoelastic Behaviors of FGM Thin-Walled Beams and Pipes Functionally Graded Structures: Aerothermoelastic Interactions Magneto-thermo-elastic Equations of Thin Electroconductive Isotropic Shells and Plates: Linear Equations 
Magneto-Thermoelastic Equations of Thin Electroconductive Isotropic

Shells and Plates: Nonlinear Equations

Thermoelastic Stability of Panels in High-Speed Flows: Linear Analysis

Thermoelastic Stability of Panels in High-Speed Flows: Nonlinear Analysis

Siti Ujila Masuri Department of Mechanical and Manufacturing Engineering, Universiti Putra Malaysia, UPM Serdang, Selangor, Malaysia

Application of GS4-1 Time Integration Framework to Linear Heat

Transfer: Transient Heat Conduction

Application of GS4-1 Time Integration Framework to Nonlinear Heat

Transfer: Heat Conduction in Medium with Temperature-Dependent

Velocity

Application of Isochronous Integration Framework to Dynamic

Thermoelasticity

Jean-Denis Mathias IRSTEA, LISC, Aubière, France

Thermal Stresses in Hybrid Composite Joints

Gerard A. Maugin Institut Jean Le Rond d'Alembert, Unité mixte de recherche 7190, Universite Pierre et Marie Curie, Paris, France

Canonical Formulation of "Nondissipative Thermoelasticity" (with

Application to Thermoelastic Fracture)

Canonical Formulation of Thermoelasticity

Classical Thermodynamics

Duhamel's Pioneering Work on Thermo-elasticity

Entropy and Thermodynamic Temperature

Internal Thermal Stresses as Quasi-Plastic Phenomena

Thermoelastic Discontinuities

Thermoelastic Fracture in Terms of Configurational Forces

Thermoelastic Shock Waves and Phase-Transition Fronts in Terms of

Configurational Forces

Thermomechanics of Continua (or Continuum Thermomechanics = CTM)

Roderick Melnik Tier I Canada Research Chair, Wilfrid Laurier University, Waterloo, ON, Canada

Soliton-Like Thermoelastic Waves

Lim Kian Meng Department of Mechanical Engineering, National University of Singapore, Singapore, Singapore

Thermal Stresses at Multi-Material Corners and Cracks in Plastic IC Packages

Craig G. Merrett Mechanical and Aerospace Engineering Department, Carleton University, Ottawa, ON, Canada

Linear Aero-Thermo-Servo-Viscoelasticity, Part I: General Theory

Linear Aero-Thermo-Servo-Viscoelasticity, Part II: Dynamic

Considerations: Lifting Surface and Panel Flutter and Aerodynamic

Noise 
M. Meshkini South Tehran Branch, Islamic Azad University, Tehran, Iran Mechanical and Thermal Stresses in a FGPM Hollow Cylinder Due to Radially Symmetric loads

Panagiotis (Pan) Michaleris Department of Mechanical and Nuclear Engineering, Pennsylvania State University, University Park, PA, USA Minimization of Welding Distortions

Welding of Large Structures

Magdalena Mierzwiczak Institute of Applied Mechanics, Poznań University of Technology, Poznań, Poland

Green's F unctions for Inverse Heat Conduction Problems

Point, Line, Surface Sources

Marine A. Mikilyan Institute of Mechanics, National Academy of Sciences of Armenia, Yerevan, Armenia

Dynamic Stability of Electroconductive Cylindrical Shells in Magnetic Field

Ferromagnetic Plates and Shells

Numerical Investigation of Magnetothermoelastic Bending of Superconductive Plate

Thermoelastic Stability of Panels in High-Speed Flows: Linear Analysis Thermoelastic Stability of Panels in High-Speed Flows: Nonlinear Analysis Vibrations of Electroconductive Cylindrical Shells in a Magnetic Field

Babak Mirzavand Faculty of New Sciences and Technologies, University of Tehran, Tehran, Iran

Thermal Buckling and Dynamic Post-Buckling Analysis of Piezoelectric FGM Hybrid Cylindrical Shells

Thermoelastic Buckling of Imperfect FGM Cylindrical Shells

Kento Mitsui Department of Mechanical Engineering, Aichi Institute of Technology, Toyota City, Aichi, Japan

Thermomechanical Properties of Shape Memory Polymer

Milan Mićunović Faculty of Technical Sciences, University of Kragujevac, Kragujevac, Serbia

Thermo-Inelastic Memory of Metals

Mamoru Mizuno Department of Machine Intelligence and Systems Engineering, Akita Prefectural University, Yuri-Honjo, Akita, Japan Piezoelectric Effects and Materials

A. H. Mohazzab Islamic Azad University, Tehran, Iran Analytical Solution for Two-Dimensional Magneto-thermomechanical Response in FG Hollow Sphere

Adriano Montanaro Department of Mathematics, Padova University, Padova, Italy

Kinematics of Singular Surfaces and Jump Equations of Balance Propagation of Wavefronts in Thermoelastic Media 
A. Moradi Postgraduate School, Islamic Azad University, South Tehran Branch, Tehran, Iran

Exact Solution for Classic Coupled Thermoelasticity in Cylindrical Coordinates

Miguel Morales Centro Láser UPM, Madrid, Spain

Induction of Thermo-Mechanical Residual Stresses in Metallic Materials by Laser Shock Processing

David G. Morris Department of Physical Metallurgy, CENIM-CSIC, Madrid, Spain

High-Temperature Creep of Iron Aluminide Intermetallics

Angelo Morro Faculty of Engineering, University of Genoa, Genoa, Italy

Functions of a Complex Variable: Differentiation and Integration

Functions of a Complex Variable: Series, Residues, and Applications

Thermoviscoelasticity and Invariance of the Entropy Production Under

Time Reversal

Vector and Tensor Analysis: Conservation Equations in Continuum Physics

Vector and Tensor Analysis: Covariant Differentiation

Vector and Tensor Analysis: Differentiation and Differential Operators

Vector and Tensor Analysis: Integral Calculus

Vector and Tensor Analysis: Singular Surfaces and Waves

Vector Spaces and Matrix Algebra: Eigenvalues and Eigenvectors

Vector Spaces and Matrix Algebra: Matrices and Determinants

Vector Spaces and Matrix Algebra: Vector and Tensor Algebra

Vector Spaces and Matrix Algebra: Vector Spaces

Zenon Mróz Institute of Fundamental Technological Research, Polish Academy of Sciences, Warsaw, Poland

Sensitivity Analysis for Thermoelastic State Fields

Sensitivity of Thermal Response with Respect to Material Parameters and Shape of Interfaces and Boundaries

Santwana Mukhopadhyay Department of Applied Mathematics, Indian Institute of Technology (Banaras Hindu University), Varanasi, India

Dual Phase-Lag Thermoelasticity

Ingo Müller Technical University Berlin, Potsdam, Germany

Thermodynamics: The Nineteenth-Century History

Dietrich Munz Forschungszentrum Karlsruhe, Karlsruhe Institute of Technology, Karlsruhe, Germany

Thermal Fatigue in Ceramics

Maria A. Muñoz-Morris Department of Physical Metallurgy, CENIMCSIC, Madrid, Spain

High-Temperature Creep of Iron Aluminide Intermetallics 
Go Murasawa Department of Mechanical Engineering, Yamagata University, Yonezawa, Yamagata, Japan

Shape Memory Alloy Composite with Short-Fiber

Gakuji Nagai Department of Mathematical and Design Engineering, Faculty of Engineering, Gifu University, Gifu City, Japan

Piezothermoelastic Analysis: Finite Element Method

Taras Nahirnyj Pidstryhach Institute for Applied Problems of Mechanics and Mathematics, National Academy of Sciences of Ukraine, Lviv, Ukraine

Local Gradient Thermomechanics

Mohammad Mahdi Najafizadeh Department of Mechanical Engineering, Islamic Azad University, Arak, Iran

Higher Order Thermal Buckling of Circular Plates

Ludovico Nappa Dipartimento di Strutture per l'Ingegneria e l'Architettura, Università degli Studi di Napoli “Federico II", Napoli, Italy

Thermal Stresses in Elastic Cylinders and Circular Shells

Thermal Stresses in Microstretch Elastic Cylinders

Variational Principles in the Theory of Elastic Bodies with Microstructure

Maria Grazia Naso Università degli Studi di Brescia, Brescia, Italy Asymptotic Behavior in Time

Existence and Uniqueness: Solutions of Thermoelastodynamics

Mathematical Models of Reissner-Mindlin Thermoviscoelastic Plates

Lucjan Nastałek Energy Conversion Department, Institute of Fluid Flow Machinery, Polish Academy of Sciences, Gdańsk, Poland

Thermodynamics of Thermo-deformable Solids

David Natroshvili Department of Mathematics, Technical University of Georgia, Tbilisi, Republic of Georgia

Boundary Value Problems of Elastostatics of Hemitropic Solids

Mathematical Problems in Thermoelastostatics of Hemitropic Solids

Thermo-radiating Conditions: Somigliana Type Integral Representations

Patrizio Neff Faculty of Mathematics, University Duisburg-Essen, Essen, Germany

Existence of Minimizers in Nonlinear Elastostatics of Micromorphic Solids

Yuriy V. Nemirovsky Institute of Theoretical and Applied Mechanics, Russian Academy of Sciences Siberian Branch, Novosibirsk, Russia Heat Conduction and Thermoelasticity of Composite Structures

Heong Wah Ng Division of Engineering Mechanics, School of Mechanical and Aerospace Engineering, Nanyang Technological University, Singapore, Singapore

Inelastic Analysis of Functionally Graded Thermal Barrier Coating (FG TBC) Under Thermal Shock 
Michel Nganbe Department of Mechanical Engineering, University of Ottawa, Ottawa, ON, Canada

Strength and Failure of High Temperature Superalloys

Yasuhiko Nishimura Department of Mechanical Engineering, Aichi Institute of Technology, Toyota City, Aichi, Japan

Reciprocating Bending Motion of Shape Memory Composite

Naotake Noda Shizuoka University, Hamamatsu, Japan

Axisymmetric Thermal Stresses in Disks

Axisymmetric Thermal Stresses in Solid Cylinders

Axisymmetric Thermal Stresses in Spheres

Moving Heat Source, Thermal Stresses

Multiply Connected Bodies, Thermal Stresses

One-Dimensional Thermal Stresses in Cylinders

Plane Thermal Stress in Cylinders

Stress Function Method for Plane Problems

Thermal Stresses in Bodies with Temperature-Dependent Properties

Thermal Stress Intensity Factors Due to Thermal Shock

Three-Dimensional Thermal Stresses in Cylinders

Serkan Nohut Faculty of Engineering, Zirve University, Gaziantep, Turkey

Effect of Thermal Stresses on Crack-Tip Toughness of Polycrystalline

Ceramics

Grzegorz Nowak Institute of Power Engineering and Turbomachinery, Silesian University of Technology, Gliwice, Poland

Design Optimization of Turbine Components with the Use of Temperature and Thermal Stresses Objectives

Władysław Nowak West Pomeranian University of Technology, Szczecin, Szczecin, Poland

Transient Heat Conduction in Semi-infinite Solid with Specified Surface Temperature

Andrzej J. Nowak Institute of Thermal Technology, Silesian University of Technology, Gliwice, Poland

Boundary Element Method in Heat Conduction

Mats Näsström Engineering Sciences and Mathematics, Luleå University of Technology, Luleå, Sweden

Welding Heat Input Models

Yoshihiro Obata Department of Mechanical and Aerospace Engineering, Graduate School of Engineering, Tottori University, Tottori, Japan

Beams, Thermal Stresses

Optimal Design of Functionally Graded Materials

José Luis Ocaña Centro Láser UPM, Madrid, Spain

Induction of Thermo-Mechanical Residual Stresses in Metallic Materials by Laser Shock Processing 
Paweł Ocłon Institute of Thermal Power Engineering, Faculty of Mechanical Engineering, Cracow University of Technology, Cracow, Poland

Finite Element Method in Steady-State and Transient Heat Conduction Hyperbolic Heat Conduction Equation

Mixed Finite Volume and Finite Element Formulation: Linear Quadrilateral Elements

Mixed Finite Volume and Finite Element Formulation: Linear Triangular Elements

Transient Heat Conduction in Sphere

Manabu Ohmichi Faculty of Engineering, Aichi University of Technology, Gamagori, Aichi, Japan

Thermal Stresses in Bars

Nobutada Ohno Graduate School of Engineering, Department of Mechanical Engineering Science, Nagoya University, Nagoya, Japan Thermal Plastic Ratcheting

Christian Olagnon INSA-Lyon MATEIS CNRS UMR5510, Villeurbanne, France

Thermal Shock and Thermal Fatigue Study of Ceramic Materials on a Newly Developed Ascending Thermal Shock Test Equipment

Mats Oldenburg Engineering Sciences and Mathematics, Luleå University of Technology, Luleå, Sweden

Warm Forming of Steels for Tailored Microstructure

Graeme John Oliver Cape Peninsula University of Science and Technology, Department of Mechanical Engineering, Cape Town, Republic of South Africa

Thermal, Mechanical, and Microstructure Couplings

Yoshihiro Ootao Department of Mechanical Engineering, Graduate School of Engineering, Osaka Prefecture University, Nakaku, Sakai, Osaka, Japan

Effect of Shear Stresses in Thermal Cylindrical Shells

Functionally Graded Cylinder

Inverse Problem of Thermal Deformation in a Cylinder

Piezothermoelastic Functionally Graded Materials

Piezothermoelastic Problems: Static and Quasi-Static Analyses

Nina Orlovskaya Department of Mechanical, Materials and Aerospace Engineering, University of Central Florida, Orlando, FL, USA Control of Thermal Residual Stresses

Norbert Ortner University of Innsbruck, Innsbruck, Austria Fundamental Solutions in Classical Thermoelasticity

Zoubeida Ounaies Department of Mechanical Engineering, The Pennsylvania State University, University Park, PA, USA Singularities of the Thermo-Magneto-Electro-Elastic Fields 
Marco Paggi Department of Structural, Geotechnical and Building Engineering, Politecnico di Torino, Torino, Italy

Thermal Contact Conductance of Rough Surfaces

Stuart J. P. Palmer SMF Fracture and Shock Physics Group, Cavendish Laboratory, University of Cambridge, Cambridge, UK

Hot Spot Ignition of Explosives and Their Thermal Properties and

Experimental Measurement of the Thermal Properties of a PBX and Its Binder System

Ernian Pan Henan Key Engineering Laboratory for Anti-fatigue Manufacturing Technology and the School of Mechanical Engineering, Zhengzhou University, Zhengzhou, People's Republic of China

Department of Civil Engineering, Computer Modeling and Simulation Group, University of Akron, Akron, OH, USA

Cracks in Transversely Isotropic and Inhomogeneous Elastic Solids

Extended Displacement Discontinuity Boundary Integral Equation Method for Analysis of Cracks in Smart Materials

Nonlinear Fracture Models of Magnetoelectroelastic Media

Andrzej Jarosław Panas Faculty of Mechatronics and Aerospace, Military University of Technology, Warsaw, Poland

Division of Aeroplanes and Helicopters, Air Force Institute of Technology, Warsaw, Poland

Moving Heat Sources

Prasanth Kumar Panda Materials Science Division, National Aerospace Laboratories, Bangalore, India

Thermal Shock and Thermal Fatigue Study of Ceramic Materials on a Newly Developed Ascending Thermal Shock Test Equipment

Ramesh Pandey Applied Mechanics Department, MNNIT, Allahabad, India

Buckling and Post-Buckling of Composite Plates Under Thermal Loads

Luc Papeleux University of Liege Aerospace and Mechanical Engineering Department, Institut de Mécanique, Liege, Belgium

Thermoviscoplasticity at Finite Strains: A Thermoelastic

Predictor-Viscoplastic Corrector Algorithm

Frederico París Group of Elasticity and Strength of Materials, School of Engineering, University of Seville, Seville, Spain

Thermal Stresses in Composite Joints

Francesca Passarella Department of Electronic and Computer Science Engineering, University of Salerno, Fisciano, SA, Italy

Spatial Behavior of Solutions

Iaroslav Pasternak Lutsk National Technical University, Lutsk, Ukraine Jump Function Method and BEM Technique for Determination of

Thermal Stresses in Solids with Thin Inclusions 
Badri Prasad Patel Department of Applied Mechanics, Indian Institute of Technology Delhi, New Delhi, India

Thermal Buckling/Postbuckling Characteristics of Laminated Composite Shells

Min Pei Intel Corporation, Chandler, AZ, USA

Thermal Stresses in Flip Chip BGA Packaging

Sandeep S. Pendhari Structural Engineering Department, Veermata Jijabai Technological Institute, Mumbai, India

High-Order Theory, Composite Plates

Thick Plates, Reissner-Mindlin Theory, Statical Problems

Dayakar Penumadu Civil and Environmental Engineering, University of Tennessee, Knoxville, TN, USA

Hygrothermal Effects on Polymeric Composite Materials and Sandwich Structures

Francesco Pesavento Department of Civil, Environmental and Architectural Engineering, University of Padova, Padova, Italy

Multiphase Porous Media, High Temperature

Bernhard J. Peters Faculté des Sciences, de la Technologie et de la Communication, Université du Luxembourg, Luxembourg, Luxembourg Energy and First Law of Thermodynamics

Giuseppe Pezzotti Ceramic Physics Laboratory \& Research Institute for Nanoscience, Kyoto Institute of Technology, Kyoto, Japan

Raman Spectroscopic Method for Analyzing Residual Stresses in Ceramic Composites

Yong-Lin Pi Centre for Infrastructure Engineering and Safety, School of Civil and Environmental Engineering, University of New South Wales, Sydney, NSW, Australia

Shallow Arches: Thermal Buckling

Daniel Pietras Faculty of Civil Engineering and Architecture, Lublin University of Technology, Lublin, Poland

Cylindrical Orthotropic Thermoelastic Shells Modeled by Direct Approach

Wojciech Pietraszkiewicz Institute of Fluid-Flow Machinery, Polish Academy of Sciences, Gdańsk, Poland

Phase Transitions in Thermoviscoelastic Shells

Jüri Pirso Department of Materials Engineering, Tallinn University of Technology, Tallinn, Estonia

Thermal Shock Resistance of Chromium Carbide-Based Cermets

Olivier Polit Laboratoire Energétique Mécanique Electromagnétisme (LEME) - EA4416, Université Paris Ouest - Nanterre - La Défense, Ville d'Avray, France

Thermal Stress Analysis of Homogeneous and Laminated Shells by Finite Element Method 
Castrenze Polizzotto Dipartimento di Ingegneria Civile, Ambientale, Aerospaziale e Materiali, Università di Palermo, Palermo, Italy

Nonlocal Elastic-Damage Models

Shakedown Under Thermomechanical Loads

Jean-Philippe Ponthot University of Liege Aerospace and Mechanical Engineering Department, Institut de Mécanique, Liege, Belgium

Thermoviscoplasticity at Finite Strains: A Thermoelastic Predictor-Viscoplastic Corrector Algorithm

Vasyl Popovych Pidstryhach Institute for Applied Problems of Mechanics and Mathematics, National Academy of Sciences of Ukraine, Lviv, Ukraine

Application of the Generalized Functions Method for Analysis of Thermal Stresses in Piecewise-Homogeneous Solids

Methods for Determination of the Thermo-stressed State of

Thermosensitive Solids Under Complex Heat Exchange Conditions

Juan Antonio Porro Centro Láser UPM, Madrid, Spain

Induction of Thermo-Mechanical Residual Stresses in Metallic Materials by Laser Shock Processing

Kevin Potter Advanced Composites Centre for Innovation and Science, University of Bristol Queen's Building, Bristol, UK

Manufacturing Thermal Stresses in Composite Components

Yuriy Povstenko Institute of Mathematics and Computer Science, Jan Długosz University, Częstochowa, Poland

Pidstryhach Institute for Applied Problems of Mechanics and Mathematics, Ukrainian National Academy of Sciences, Lviv, Ukraine

Fractional Thermoelasticity

G. Pranckevicius Institute of Physical and Technical Problems of Energetic, Vilnius, Lithuania

Heating Methods of Testing for Thermal Stress Resistance

William Prince Civil Engineering Department, INSA de Rennes, Rennes, France

Damage in Granite Under Temperature Variations

Borys Protsiuk Pidstryhach Institute for Applied Problems of Mechanics and Mathematics, National Academy of Sciences of Ukraine, Lviv, Ukraine

Determination of the Thermal Fields and Stresses in Multilayer Solids by Means of the Constructed Green Functions

Xuele Qi Department of Mechanical Engineering, Texas A\&M University, College Station, TX, USA

Temperature Characterization in Silicon Wafer by Using Stress Wave as an Indicator 
Thermoelastodynamics for Silicon Materials Subjected to Ultrafast Laser Heating

Ultrafast Laser-Induced Thermoelastic Responses in Semiconductors

Chen Qian College of Aerospace Engineering, Institute of Vibration Engineering Research, Nanjing University of Aeronautics and Astronautics, Nanjing, People's Republic of China

Aerothermoelastic Control of Lifting Surfaces

Qing-Hua Qin Research School of Engineering, The Australian National University, Canberra, ACT, Australia

General Solutions for Thermopiezoelectric Materials with Various Openings

Green's Functions of Magneto-Electro-Elastic Plate Under Thermal Loading

Zhanming Qin State Key Lab for Strength and Vibration of Mechanical Structures, Xi'an Jiaotong University, Xi' an, People's Republic of China

Electroconductive Composites Subjected to Magnetoelastic and Thermomechanical Loadings: Nonlinear Response and Stability

Magneto-thermo-elastic Equations of Thin Electroconductive Isotropic Shells and Plates: Linear Equations

Magneto-Thermoelastic Equations of Thin Electroconductive Isotropic Shells and Plates: Nonlinear Equations

Magneto-Thermo-Elasticity of Laminated Composite Plates Incorporating Structural Nonlinearity

Ramon Quintanilla Matemática Aplicada 2, Universitat Politècnica de Catalunya, Terrassa, Barcelona, Spain

Continuous Data Dependence in Linear Theories of Thermoelastodynamics. Part I: Classical Theories. Basics and Logarithmic Convexity

Continuous Data Dependence in Linear Theories of Thermoelastodynamics. Part II: Classical Theories, Lagrange Identity Methods, and Positive-Definite Arguments

Continuous Data Dependence in Linear Theories of Thermoelastodynamics. Part III: Nonclassical Theories Spatial and Structural Stability in Thermoelastostatics

G. Venkateswara Rao Department of Mechanical Engineering, Vardhaman College of Engineering, Hyderabad, Andhra Pradesh, India Post-Buckling of Heated Circular Plates

Mongi Rekik Applied Mechanics and Systems Research Laboratory, Tunisia Polytechnic School, University of Carthage, La Marsa, Tunisia Singularities of the Thermo-Magneto-Electro-Elastic Fields

Nicolás M. Rendtorff Centro de Tecnología de Recursos Minerales y Cerámica (CETMIC), (CIC-CONICET-CCT La Plata), Buenos Aires, Argentina 
Facultad de Ciencias Exactas - Universidad Nacional de La Plata, UNLP, Argentina

CIC-PBA, Buenos Aires, Argentina

Thermal Shock Resistance (TSR) and Thermal Fatigue Resistance (TFR) of Refractory Materials. Evaluation Method Based on the Dynamic Elastic Modulus

Matti Ristinmaa Division of Solid Mechanics, Lund University, Lund, Sweden

Isotropic/Kinematic Hardening in Thermoplasticity

Abdel-Fattah Rizk Department of Engineering Mathematics and Physics, Alexandria University, Alexandria, Egypt

Edge Crack, Bimaterial Systems

Edge Crack, Composite Materials

Edge Crack, Isotropic Material

Marcello Romagnoli Dipartimento di Ingegneria "Enzo Ferrari", Università degli Studi di Modena e Reggio Emilia, Modena, Italy

New Methods for the Assessment of Thermal Shock Resistance in

Refractory Materials

Jacek Ronda Faculty of Metals Engineering and Industrial Computer Science, AGH University of Science and Technology, Cracow, Poland

Numerical Modelling of Underwater Welding and Cutting

Thermal, Mechanical, and Microstructure Couplings

Yury A. Rossikhin Research Center on Wave Dynamics in Solids and Structures, Voronezh State University of Architecture and Civil Engineering, Voronezh, Russia

Boundary-Value Problems Resulting in Thermoelastic Shock Wave Propagation

D'Alembert Method in Dynamic Problems of Thermoelasticity

Harmonic Waves in an Anisotropic Medium Generated by Heat Sources Distributed Along the Plane

Hyperbolic Thermoelasticity, Transient Dynamic Contact Problems

Ray Expansion Theory

Ray Method for Solving Boundary-Value Problems of Anisotropic

Thermoelasticity with Thermal Relaxation

Thermal Shock upon Thin-Walled Beams of Open Profile

Transient Thermoelastic Rayleigh Waves on the Surfaces of Bodies of Revolution

Hessam Rouhi Department of Mechanical Engineering, University of Guilan, Rasht, Iran

Thermal Buckling of Carbon Nanotubes

M. Rozniakowska-Klosinska Technical University of Łódź, Łódź, Poland Laser-Induced Thermal Splitting in Homogeneous Body with Coating 
Gregory Rudin Department of Mechanical Engineering, Pearlstone Center for Aeronautical Engineering Studies, Ben-Gurion University of the Negev, Beer-Sheva, Israel

Thermal Stress Caused by Laser Pulse in Functionally Graded Materials

Leonardo Ruiz de Lara Centro Láser UPM, Madrid, Spain

Induction of Thermo-Mechanical Residual Stresses in Metallic Materials by Laser Shock Processing

Andrzej Rusin Institute of Power Engineering and Turbomachinery, Silesian University of Technology, Gliwice, Poland

Design Optimization of Turbine Components with the Use of Temperature and Thermal Stresses Objectives

Transient Temperature and Thermal Stresses in Turbine Components

Remigio Russo Department of Mathematics and Physics, Second University of Naples, Caserta, Italy

Classical Coupled Thermoelasticity in Unbounded Domains

Linear Elastodynamics in Unbounded Domains

Uniqueness in Linear Elastostatics

Suk-Kyu Ryu Department of Aerospace Engineering and Engineering Mechanics, University of Texas, Austin, TX, USA

Thermal Stress in 3-D Packaging

Ibrahim S. Sadek Faculty of Civil Engineering and Architecture, Lublin University of Technology, Lublin, Poland

Piezoelectric Actuation: Control of Linear Thermal Vibrations

Tomasz Sadowski Faculty of Civil Engineering and Architecture, Lublin University of Technology, Lublin, Poland

Cylindrical Orthotropic Thermoelastic Shells Modeled by Direct Approach

Ömer Sinan Şahin Department of Mechanical Engineering, Selcuk University, Konya, Turkey

Thermal Buckling of Plates with Hole

Akihide Saimoto Graduate School of Engineering, Nagasaki University, Nagasaki, Japan

Body Force Method for Thermoelasticity

Control of Crack Propagation

Sei-Ichiro Sakata Department of Mechanical Engineering, Faculty of Science and Engineering, Kinki University, Higashi-Osaka, Osaka, Japan

Optimization of Thermoelastic Structures

Ali Sangghaleh Department of Civil Engineering, Computer Modeling and Simulation Group, University of Akron, Akron, OH, USA

Cracks in Transversely Isotropic and Inhomogeneous Elastic Solids 
Ivan Saprunov Center for Experimental Mechanics, Faculty of Mechanical Engineering, University of Ljubljana, Ljubljana, Slovenia Experimental Determination of Material Time-Dependent Properties

Radijanka Savova Bulgarian Academy of Sciences, Sofia, Bulgaria Surface Wave Propagation in a Thermoelastic Half-Space

Antonio Scalia Department of Mathematics and Computer Science, University of Catania, Catania, Italy

Basic Theorems in Thermoelastostatics of Bodies with Microtemperatures

Deformation of Anisotropic Elastic Cylinders with Microstructure Representations of Solutions in Thermoelasticity Theory

Gary S. Schajer Department of Mechanical Engineering, University of British Columbia, Vancouver, Canada

Hole-Drilling Residual Stress Measurements

Przemyslaw Scherwentke Faculty of Fundamental Problems of Technology, Institute of Mathematics and Computer Science, Wrocław Technical University, Wrocław, Poland

Selected Problems of Elastostatics and Thermoelastostatics of the

Hemitropic Micropolar Medium

Bernhard A. Schrefler Department of Civil, Environmental and Architectural Engineering, University of Padova, Padova, Italy

Multiphase Porous Media, High Temperature

Maximilian Schwenk Institute of Applied Materials, Institute of Technology, Karlsruhe, Germany

Transformation Hardening in Steel

Nigel H. Scott School of Mathematics, University of East Anglia, Norwich, UK

Acceleration Wave Propagation in Inhomogeneous Heat-Conducting Rods

Inhomogeneous Plane Waves in Thermoelasticity

Antonio Sellitto Department of Mathematics, Computer Science and Economics, University of Basilicata, Potenza, Italy

Nonequilibrium Temperatures and Second-Sound Propagation Along Nanowires

Igor K. Senchenkov Timoshenko Institute of Mechanics, National Academy of Sciences of Ukraine, Kiev, Ukraine Modeling of Thermomechanical Process in Growing Viscoplastic Bodies with Accounting of Microstructural Transformation

Miroslaw Seredynski Institute of Heat Engineering, Warsaw University of Technology, Warsaw, Poland Phase Change Heat Transfer Problems 
Victor Seremet Laboratory of Mathematical Modeling, Institute of Mathematics and Computer Science, Academy of Science of Moldova, Chisinau, MD, Moldova

Laboratory of Green's Functions, Agrarian State University of Moldova, Chisinau, MD, Moldova

Green's Functions in Three-Dimensional Thermoelastostatics

Igor L. Shabalin Materials \& Physics Research Centre, University of Salford, Salford, Greater Manchester, UK

TSR of Hetero-modulus Ceramics

Ali Shakouri Department of Electrical Engineering, University of California, Santa Cruz, CA, USA

Thermal Stress in a Multi-leg Thermoelectric Module (TEM) Design

Dongkai Shangguan Flextronics Corporation, Milpitas, CA, USA

Thermal Stresses in a Tri-Material Assembly with Application to Silicon-Based Photovoltaic Module (PVM)

N. Shankar Department of Mechanical Engineering, University of Minnesota, Minneapolis, MN, USA

Department of Mechanical Engineering, National Institute of Technology Karnataka, Surathkal Srinivasnagar, KA, India

Mechanical Engineering and Engineering Mechanics, Michigan Technological University, Houghton, MI, USA

Equation of Phonon Radiative Transport: Formulation and Analysis by the Weighted Residual Method

J. N. Sharma Department of Mathematics, National Institute of Technology, Hamirpur, India

Lamb Waves in Homogeneous Isotropic Thermoelastic Plates

Thermoelastic Waves, Homogeneous Isotropic Plates

Wave Propagation in Coupled and Generalized Thermoelastic Media

M. D. Sharma Department of Mathematics, Kurukshetra University, Kurukshetra, India

Wave-Field in Anisotropic Thermoviscoelastic Media

Leon L. Shaw Department of Mechanical, Materials and Aerospace Engineering, Illinois Institute of Technology, Chicago, IL, USA

Thermal Stress Modeling in Multi-Material Laser Processing

Hui-Shen Shen Department of Engineering Mechanics, Shanghai Jiao Tong University, Shanghai, People's Republic of China

Thermoelastic Buckling and Postbuckling of Plates on Elastic Foundations

M. H. Shen Department of Automation Engineering, Nan Kai University of Technology, Tsao Tun, Nantou, Taiwan, Republic of China

Curvilinear Cracks

Interaction Between a Heat Source and a Circular Inclusion with a SemiInfinite Crack 
Yapeng Shen MOE Key Laboratory of Strength \& Vibration, Department of Engineering Mechanics, Xi' an Jiaotong University, Xi'an, Shannxi Province, People's Republic of China

Piezothermoelasticity with Finite Wave Speeds

Yu-Lin Shen Department of Mechanical Engineering, University of New Mexico, Albuquerque, NM, USA

Material Properties of Electronic Packages, Thermal Stresses in On-Chip Metal Interconnects

R. Ajit Shenoi School of Engineering Sciences, University of Southampton, Southampton, UK

Residual Thermal Stress in 2D Orthogonal Plain Weave Fabric Composites

Hany H. Sherief Department of Mathematics, Faculty of Science, University of Alexandria, Alexandria, Egypt

Axisymmetric Generalized Thermoelasticity Problems in Spherical Regions

Boundary Element Method in Generalized Thermoelasticity

State-Space Approach to Generalized Thermoelasticity

Yuriy Shevchenko Timoshenko Institute of Mechanics, National Academy of Sciences of Ukraine, Kiev, Ukraine

Computational Methods in Stationary and Nonstationary ThermalPlasticity Problems

Victor A. Shevchuk Pidstryhach Institute for Applied Problems of Mechanics and Mathematics, National Academy of Sciences of Ukraine, Lviv, Ukraine

Generalized Boundary Conditions to Solving Thermal Stress Problems for Bodies with Thin Coatings

Meir Shillor Department of Mathematics and Statistics, Oakland University, Rochester, MI, USA

Thermoelastic Contact, Rod Models

Thermoelastic Stresses, Variational Methods

M. Shimada Department of Mechanical Engineering, University of Minnesota, Minneapolis, MN, USA

Equation of Motion: Scalar Formalisms and Theory

Equation of Motion: Vector Formalism and Theory

Explicit Time Integrators and Designs for First-/Second-Order Linear Transient Systems

Implicit Time Integrators and Designs for First-/Second-Order Linear Transient Systems

Implicit Time Integrators and Designs for Nonlinear Second-Order Systems: N-Body Systems

Implicit Time Integrators and Designs for Nonlinear Second-Order Transient Systems: Elastodynamics 
Marina V. Shitikova Research Center on Wave Dynamics in Solids and Structures, Voronezh State University of Architecture and Civil Engineering, Voronezh, Russia

Boundary-Value Problems Resulting in Thermoelastic Shock Wave Propagation

D'Alembert Method in Dynamic Problems of Thermoelasticity

Harmonic Waves in an Anisotropic Medium Generated by Heat Sources Distributed Along the Plane

Hyperbolic Thermoelasticity, Transient Dynamic Contact Problems

Ray Expansion Theory

Ray Method for Solving Boundary-Value Problems of Anisotropic

Thermoelasticity with Thermal Relaxation

Thermal Shock upon Thin-Walled Beams of Open Profile

Transient Thermoelastic Rayleigh Waves on the Surfaces of Bodies of Revolution

Amir Shojaei Department of Mechanical Engineering, Louisiana State University, Baton Rouge, LA, USA

Thermal Cyclic Loading of Beams Based on the Chaboche Kinematic Hardening Model

Arun Shukla Dynamics Photomechanics Laboratory, Department of Mechanical, Industrial and Systems Engineering, University of Rhode Island, Kingston, RI, USA

Performance of Sandwich Structures Under Dynamic Loads at Different Temperatures

Karunesh Kumar Shukla Applied Mechanics Department, MNNIT, Allahabad, India

Buckling and Post-Buckling of Composite Plates Under Thermal Loads

Roman Shvets Pidstryhach Institute for Applied Problems of Mechanics and Mathematics, National Academy of Sciences of Ukraine, Lviv, Ukraine

Thermoelasticity of Thin Shells

Jaan Simon Institut für Angewandte Mechanik, RWTH Aachen University, Aachen, Germany

Limit States and Failure of Elastoplastic Structures Under Thermomechanical Loading

Baljeet Singh Department of Mathematics, Post Graduate Government College, Chandigarh, India

Thermoelastic Waves at an Interface Between Two Solids

Akawut Siriruk Civil and Environmental Engineering, University of Tennessee, Knoxville, TN, USA

Hygrothermal Effects on Polymeric Composite Materials and Sandwich Structures 
Błażej Skoczeń Institute of Applied Mechanics, Faculty of Mechanical Engineering, Cracow University of Technology, Cracow, Poland

Cryogenic Condition, Damage In

Discontinuous Yielding at Cryogenic Temperatures

Ductile Damage at Cryogenic Temperatures

Jacek Skrzypek Institute of Applied Mechanics, Cracow University of Technology, Cracow, Poland

Anisotropic Initial Yield and Failure Criteria Including Temperature Effect

Jan Sladek Department of Mechanics, Institute of Construction and Architecture, Slovak Academy of Sciences, Bratislava, Slovakia Application of Boundary Integral Equation (BIE) Method in

Thermoelastodynamic Problem

Vladimir Sladek Department of Mechanics, Institute of Construction and Architecture, Slovak Academy of Sciences, Bratislava, Slovakia Application of Boundary Integral Equation (BIE) Method in Thermoelastodynamic Problem

V. Slyunyayev Institute for Problems of Materials Science, Kiev, Ukraine Control of Thermal Residual Stresses

Mike Smith EDF Energy Nuclear Generation Ltd, Gloucester, UK Modeling of Welding of Austenitic Stainless Steels

Tomasz Sobota Institute of Thermal Power Engineering, Faculty of Mechanical Engineering, Cracow University of Technology, Cracow, Poland Energetics

Fourier's Law of Heat Conduction

General Heat Conduction Equation in Various Coordinate Systems

Abdullah Sofiyev Department of Civil Engineering, Suleyman Demirel University, Isparta, Turkey

FGM Cones Surrounded by Pasternak-Type Elastic Medium Subjected to Thermal Load

Saeed Sohrabpour Mechanical Engineering Department, Sharif University of Technology, Tehran, Iran

Mechanical and Thermal Stresses in a Functionally Graded Hollow Cylinder Due to Nonaxisymmetric Steady-State Loads

Mechanical and Thermal Stresses in a Functionally Graded Hollow Cylinder Due to Radially Symmetric Loads

Marta Solecka Faculty of Civil Engineering, Architecture and Environmental Engineering, Technical University of Łódź, Łódź, Poland

Generalized Stochastic Perturbation-Based Finite Element Analysis of Structures 
Fan Song State Key Laboratory of Nonlinear Mechanics (LNM), Institute of Mechanics, Chinese Academy of Sciences, Beijing, People's Republic of China

Enhanced Thermal Shock Resistance of Ceramics

Horacio Sosa Rowan University, Glassboro, NJ, USA

Piezothermoelasticity: Fundamental Equations

Abdelaziz Soufyane Faculty of Engineering and Applied Sciences, ALHOSN University, Abu Dhabi, United Arab Emirates

Porous Thermoelasticity with Applications

Hari M. Srivastava Department of Mathematics and Statistics, University of Victoria, Victoria, BC, Canada

Special Functions

Laurent Stainier Research Institute of Civil and Mechanical Engineering (GeM, UMR 6183 CNRS), Ecole Centrale Nantes, Nantes, France Mechanically Generated Heat

Giulio Starita Department of Mathematics and Physics, Second University of Naples, Caserta, Italy

Uniqueness in Linear Elastostatics

Pavlo Steblyanko Dneprodzerzhinsk State Technical University, Dneprodzerzhinsk, Ukraine

Computational Methods in Stationary and Nonstationary Thermal-Plasticity Problems

Brian Straughan Department of Mathematical Sciences, Science Laboratories, Durham University, Durham, UK

Acceleration Waves in Thermoelastic Materials with Voids

Some Remarks on Functional Analysis

Dmitry Strunin Department of Mathematics and Computing, University of Southern Queensland, Toowoomba, QLD, Australia

Soliton-Like Thermoelastic Waves

Yoshihiro Sugano Department of Mechanical Systems Engineering, Iwate University, Morioka, Iwate, Japan

Stochastic Analysis of Thermal Stresses in Bodies

C. Steve Suh Department of Mechanical Engineering, Texas A\&M University, College Station, TX, USA

Temperature Characterization in Silicon Wafer by Using Stress Wave as an Indicator

Thermoelastodynamics for Silicon Materials Subjected to Ultrafast Laser Heating

Ultrafast Laser-Induced Thermoelastic Responses in Semiconductors 
Ephraim Suhir Department of Electrical Engineering, University of California, Santa Cruz, CA, USA

Analysis of a Prestressed Bi-Material Accelerated-Life-Test (ALT)

Specimen

Thermal Stress in a Multi-leg Thermoelectric Module (TEM) Design

Thermal Stresses in a Tri-Material Assembly with Application to Silicon-

Based Photovoltaic Module (PVM)

Thermoelastic Stability of an Embedded Nano-fiber

Heorhiy Sulym Ivan Franko National University of Lviv, Lviv, Ukraine

Jump Function Method and BEM Technique for Determination of

Thermal Stresses in Solids with Thin Inclusions

Naobumi Sumi Faculty of Education, Shizuoka University, Shizuoka, Japan

Characteristic Method of Thermal Stresses

Moving Heat Source, Thermal Stresses

Multiply Connected Bodies, Thermal Stresses

Orthotropic Rectangular Plate with a Rigid Ribbonlike Inclusion, Thermal Stress

Wei Sun Department of Mechanical, Materials and Manufacturing Engineering, University of Nottingham, Nottingham, UK

Finite Element Simulation of the Fusion Welding of Metal Components Including Post-weld Heat Treatment

Mehmet Sunar Mechanical Engineering Department, King Fahd University of Petroleum \& Minerals, Dhahran, Saudi Arabia

Piezoelectric Sensors for Application to Thermoelastic Structures

Piezoelectric Smart Structures for Control of Thermoelastic Response

Manfred Suppa Lackwerke Peters GmbH+Co KG, Kempen, Germany Reliability of Photoimageable Solder Resists for Electronic Assemblies when Exposed to Loads Under Continuous Thermal Stress and Thermal Shock Stress

Merab Svanadze Institute for Fundamental and Interdisciplinary Mathematics Research, Ilia State University, Tbilisi, Georgia

Basic Theorems in Thermoelastostatics of Bodies with Microtemperatures

Fundamental Solutions in Thermoelasticity Theory

Fundamental Solutions in Thermoelastostatics of Micromorphic Solids

Large Existence of Solutions in Thermoelasticity Theory of Steady

Vibrations

Potentials in Thermoelasticity Theory

Representations of Solutions in Thermoelasticity Theory

Andras Szekeres Department Applied Mechanics, Budapest University of Technology and Economics, Budapest, Hungary

Thermo-Hygro-Elasticity (THE) 
Mokhfi Takarli Civil Engineering Department, Université de Limoges, Limoges, France

Damage in Granite Under Temperature Variations

Kohei Takeda Department of Mechanical Engineering, Aichi Institute of Technology, Toyota City, Aichi, Japan

Subloop Deformation Properties of Shape Memory Alloy

Lakhdar Taleb Material Physics Group, Institut National des Sciences Appliquées, Rouen, France

Transformation-Induced Plasticity (TRIP)

Giorgio Talenti University of Florence, Florence, Italy

Integral Equations

Dawid Taler Institute of Engineering and Air Protection, Faculty of Environmental Engineering, Cracow University of Technology, Cracow, Poland Finite Volume Method in Heat Conduction

Fins of Rectangular and Hexagonal Geometry

Fins of Straight and Circular Geometry

Optimum Heating of Thick Plane Wall

Superposition Method for Time-Dependent Thermal Boundary Conditions Surface-Heat Transfer Measurements Using Transient Techniques

Jan Taler Institute of Thermal Power Engineering, Faculty of Mechanical Engineering, Cracow University of Technology, Cracow, Poland Exact Solution of Inverse Heat Conduction Problems

Finite Element Method in Steady-State and Transient Heat Conduction Mixed Finite Volume and Finite Element Formulation: Linear Quadrilateral Elements

Mixed Finite Volume and Finite Element Formulation: Linear Triangular Elements

Monitoring of Thermal Stresses in Pressure Components of Steam Boilers Optimum Heating of Thick Plane Wall

Space- and Time-Marching Methods for One-Dimensional Inverse Heat

Conduction Problems

Space-Marching Method for Two-Dimensional Inverse Heat Conduction Problems

Superposition Method for Multidimensional Heat Conduction Problems Superposition Method for Time-Dependent Thermal Boundary Conditions Surface-Heat Transfer Measurements Using Transient Techniques Transient Heat Conduction in Sphere

Kumar K. Tamma Department of Mechanical Engineering, University of Minnesota, Minneapolis, MN, USA

Application of GS4-1 Time Integration Framework to Linear Heat

Transfer: Transient Heat Conduction

Application of GS4-1 Time Integration Framework to Nonlinear Heat

Transfer: Heat Conduction in Medium with Temperature-Dependent Velocity 
Application of Isochronous Integration Framework to Dynamic

Thermoelasticity

Ballistic-Diffusive Approximation: A New Look

$C$ - and F-Processes Model and Dynamic Thermoelasticity

C-and F-Processes Model: A Generalized Approach to Solving Transient

Diffusive, Wavelike and Ballistic Solid State Heat Conduction Problems

Classical Thermomechanical Models: Numerical Formulations

Classical Thermomechanical Models: Theoretical Formulations

Equation of Motion: Scalar Formalisms and Theory

Equation of Motion: Vector Formalism and Theory

Equation of Phonon Radiative Transport: Formulation and Analysis by the

Weighted Residual Method

Explicit Time Integrators and Designs for First-/Second-Order Linear

Transient Systems

Heat Conduction with Phase Change: Multiple Phase Fronts and Time-

Step Adaptation-2

Heat Conduction with Phase Change: Theoretical and Computational

Formulations-1

Implicit Time Integrators and Designs for First-/Second-Order Linear

Transient Systems

Implicit Time Integrators and Designs for Nonlinear Second-Order Systems: N-Body Systems

Implicit Time Integrators and Designs for Nonlinear Second-Order

Transient Systems: Elastodynamics

Macroscale One Temperature Theory: Heat Transfer and Constitutive

Models

Microscale Two-Temperature Theory: Heat Transfer and Constitutive

Models

Nonclassical Thermomechanical Models: Numerical Formulations

Nonclassical Thermomechanical Models: Theoretical Formulations

Thermal Contact Applications: Finite Element Formulations

Thermal Contact Applications: Mechanical Contact Models

Thermal Contact Applications: Thermal Contact Models

Transient Thermal Analysis: The Virtual-Pulse Time Integral Method

Yoshinobu Tanigawa Osaka Prefecture University, Osaka, Japan

Analytical Method of FGM

Axisymmetric Thermal Stresses in Spheres

David W. J. Tanner Department of Mechanical, Materials and Manufacturing Engineering, University of Nottingham, Nottingham, UK

Finite Element Simulation of the Fusion Welding of Metal Components

Including Post-weld Heat Treatment

Kazumi Tanuma Department of Mathematics, School of Science and Technology, Gunma University, Kiryu, Japan

Stroh Formalism for Elastostatics of Anisotropic Solids 
Dimitriy V. Tarlakovskii State University of Aerospace Technologies, Moscow, Russia

Dynamic Processes in Thermo-Electro-Magneto-Elastic and ThermoElasto-Diffusive Media

Method of Asymptotic Separation of Variables in Problems of Thermoelasticity

Method of Averaging in Problems of Thermoelasticity of Composite Materials

Theodore R. Tauchert Department of Mechanical Engineering, University of Kentucky, Lexington, KY, USA

Circular Plates, Statical Problems

Energy Method, Anisotropic and Heterogeneous Plates

Energy Method, Homogeneous Isotropic Plates

Identification of Thermo-microstretch Moduli of Materials by the Use of Vibrational Data of Plates

Laminated Plates, Antisymmetric and Nonsymmetric

Laminated Plates, Symmetric

Large Plate Deflections, Berger's Approximation

Large Plate Deflections, von Kármán Theory, Dynamical Problems

Large Plate Deflections, von Kármán Theory, Statical Problems

Maysel's Method for Plates

Plates with Temperature-Dependent Properties

Plates, Anisotropic and Heterogeneous

Plates, Classical Theory

Rectangular Plates, Statical Problems

Stability, Heterogeneous Anisotropic Plates

Thermal Buckling, Homogeneous Isotropic Plates

Thermally Induced Vibration, Isotropic Plates

Thermally Induced Vibration, Orthotropic Plates

Thick Plates, Energy Method

Andrew Tay Department of Mechanical Engineering, National University of Singapore, Singapore, Singapore

Thermal Stresses at Multi-Material Corners and Cracks in Plastic IC Packages

Kostiantyn Tchervinka Ivan Franko National University of Lviv, Lviv, Ukraine

Local Gradient Thermomechanics

Vasco Teixeira Department of Physics, School of Sciences, University of Minho, Guimarães, Portugal

Residual Stresses in Thin Films Evaluated by Different Experimental Techniques

Janusz Terpiłowski Faculty of Mechatronics, Military University of Technology, Warsaw, Poland

Impulse Method for Determining Thermal Diffusivity of Solids 
Ole Thybo Thomsen Department of Mechanical and Manufacturing Engineering, Aalborg University, Aalborg East, Denmark

Thermomechanical Nonlinear Response of Sandwich Panels

Xiaogeng Tian MOE Key Laboratory of Strength \& Vibration, Department of Engineering Mechanics, Xi'an Jiaotong University, Xi'an, Shannxi Province, People's Republic of China

Piezothermoelasticity with Finite Wave Speeds

Vincenzo Tibullo Dipartimento di Ingegneria Elettronica e Ingegneria Informatica, University of Salerno, Fisciano, SA, Italy

Harmonic Vibrations in Thermoelastic Cylinders

Spatial Behavior Backward in Time

Hisaaki Tobushi Department of Mechanical Engineering, Aichi Institute of Technology, Toyota City, Aichi, Japan

Reciprocating Bending Motion of Shape Memory Composite

Subloop Deformation Properties of Shape Memory Alloy

Thermomechanical Properties of Shape Memory Polymer

Keiichiro Tohgo Department of Mechanical Engineering, Shizuoka University, Hamamatsu, Shizuoka, Japan

Shape Memory Alloy Composite with Short-Fiber

Yuriy V. Tokovyy Pidstryhach Institute for Applied Problems of Mechanics and Mathematics, National Academy of Sciences of Ukraine, Lviv, Ukraine Direct Integration Method Nonhomogeneous Solids: Integral Equation Approach

Jalal Torabi Department of Mechanical Engineering, Amirkabir University of Technology, Tehran, Iran

Linear Thermal Buckling of Truncated FGM Conical Shells

Linear Thermal Buckling of Truncated Isotropic Conical Shells with Piezoelectric Layers

Marian Trela Institute of Fluid-Flow Machinery, Polish Academy of Sciences, Gdańsk, Poland

Thermodynamics of Machines

Marcin Trojan Institute of Thermal Power Engineering, Faculty of Mechanical Engineering, Cracow University of Technology, Cracow, Poland One-Dimensional, Steady-State Heat Conduction

Transient Heat Conduction in Semi-infinite Solid with Surface Convection

Tomoaki Tsuji Chuo University, Kasuga, Bunkyouku, Tokyo, Japan Molecular Dynamics Method for Thermoelasticity

Cem. C. Tutum Department of Mechanical Engineering, Section of Manufacturing Engineering, Technical University of Denmark, Lyngby, Denmark

Modeling Residual Stresses in Friction Stir Welding of Al Alloys 
Sei Ueda Department of Mechanical Engineering, Osaka Institute of Technology, Osaka, Japan

Infinite Row of Parallel Cracks in a Piezoelectric Material Strip

Piezoelectric Materials with Multi-Cracks

Yasutomo Uetsuji Department of Mechanical Engineering, Osaka Institute of Technology, Osaka, Japan

Piezothermoelastic Analysis: Homogenization Modeling

Giovanna Valenti Department of Mathematics and Informatics, University of Messina, Messina, Italy

Acceleration Waves in Layers of Isotropic Solids at Finite Temperatures

Murilo Augusto Vaz Ocean Engineering Program, Federal University of Rio de Janeiro, Rio de Janeiro, RJ, Brazil

Thermal Buckling of Beams on Elastic Foundation

Thermomechanical Inelastic Buckling of Beams Resting on an Elastic Foundation

Arnold Verruijt Faculty of Civil Engineering and Geosciences, Delft University of Technology, Delft, The Netherlands

Elastostatics of a Half Space

V. A. Vestyak State University of Aerospace Technologies, Moscow, Russia

Dynamic Processes in Thermo-Electro-Magneto-Elastic and Thermo-Elasto-Diffusive Media

Method of Asymptotic Separation of Variables in Problems of Thermoelasticity

Method of Averaging in Problems of Thermoelasticity of Composite Materials

D. Vicentini Group of Elasticity and Strength of Materials, School of Engineering, University of Seville, Seville, Spain

Thermal Stresses in Composite Joints

Philippe Vidal Laboratoire Energétique Mécanique Electromagnétisme (LEME) - EA4416, Université Paris Ouest - Nanterre - La Défense, Ville $\mathrm{d}^{\prime}$ Avray, France

Thermal Stress Analysis of Homogeneous and Laminated Shells by Finite Element Method

Dwight Viehland Department of Material Science and Engineering, Virginia Polytechnic Institute and State University, Blacksburg, VA, USA

Electroconductive Composites Subjected to Magnetoelastic and Thermomechanical Loadings: Nonlinear Response and Stability Magneto-Thermoelastic Equations of Thin Electroconductive Isotropic Shells and Plates: Nonlinear Equations

Thermo-Magneto-Electro-Elastic Multilayer Composites: Effective Properties and Magneto-electric Coefficients 
Josef Voldřich New Technologies - Research Centre, University of West Bohemia, Pilsen, Czech Republic

Brakes, Thermal and Thermoelastic Analysis

Milena Vujosevic Intel Corporation, Folsom, CA, USA

Thermally Induced Deformations in Assembly of FCBGA Packages

Peter Wagner University of Innsbruck, Innsbruck, Austria

Fundamental Solutions in Classical Thermoelasticity

Piotr Wais Institute of Thermal Power Engineering, Faculty of Mechanical Engineering, Cracow University of Technology, Cracow, Poland

Explicit Finite-Difference Method for Solving Transient Heat Conduction Problems

Extended Surfaces (Fins and Pins)

Implicit Finite-Difference Method for Solving Transient Heat Conduction Problems

Stephen M. Walley SMF Fracture and Shock Physics Group, Cavendish Laboratory, University of Cambridge, Cambridge, UK

Hot Spot Ignition of Explosives and Their Thermal Properties and

Experimental Measurement of the Thermal Properties of a PBX and Its Binder System

Baolin Wang Center for Composite Materials, Harbin Institute of Technology, Harbin, People's Republic of China

Piezoelectric Materials with Electrodes

Piezoelectric Materials with Penny-Shaped Crack

Chung-Hao Wang Aeronautical Department, National Formosa University, Yunlin, Taiwan, Republic of China

Perturbation Solutions of Temperature Distribution of a Plane with a Nearly Circular Inclusion

Huiming Wang Department of Engineering Mechanics, Zhejiang University, Hangzhou, People's Republic of China

Piezothermoelastic Problems: Dynamic Analyses

Ji Wang Piezoelectric Device Laboratory, School of Mechanical Engineering \& Mechanics, Ningbo University, Ningbo, Zhejiang, People's Republic of China High-Frequency Vibrations of Piezoceramic Plates

Surface Acoustic Waves in Finite Piezoceramic Solids

Min-Zhong Wang Department of Mechanics and Aerospace Engineering, State Key Laboratory for Turbulence and Complex Systems, College of Engineering, Peking University, Beijing, People's Republic of China

Stroh Formalism

Xian Feng Wang College of Civil Engineering, Shenzhen University, Shenzhen, Guangdong, People's Republic of China

Interaction Between a Rigid Inclusion and a Line Crack Under Uniform Heat Flux 
Xin Wang Department of Materials, Imperial College, London, UK

Photoluminescence Piezo-Spectroscopy Method for Measurement of Residual Stresses

Xinwei Wang Department of Mechanical Engineering, Iowa State University, Ames, IA, USA

Stresses in Laser Surface Nanostructuring

Thermoelastic Waves Induced by Pulsed Laser Heating

Ya-Guang Wang Department of Mathematics, and MOE-LSC, Shanghai Jiao Tong University, Shanghai, People's Republic of China

Global Existence and Exponential Stability in Nonlinear

Thermoelasticity

Propagation of Singularities

Yuequan Wang College of Material Science and Technology, Nanjing University of Aeronautics and Astronautics, Nanjing, People's Republic of China Laminated Plates, Interlaminar Stresses

Yunda Wang DARPA Center for Integrated Micro/Nano-Electromechanical Transducers (iMINT), Department of Mechanical Engineering, University of Colorado, Boulder, CO, USA

Thermal Stress in MEMS

Chen Wanji Key Laboratory of Liaoning Province for Composite Structural Analysis of Aerocraft and Simulation, Shenyang Aerospace University, Shenyang, People's Republic of China

High-Order Theory, Laminated Plate Buckling

Laminated Plates, Hygrothermal Effects

Kazumi Watanabe Department of Mechanical Engineering, Yamagata University, Yonezawa, Yamagata, Japan

Thermoelastic Green's Function for an Inhomogeneous Solid

Bohdan Weglowski Institute of Thermal Power Engineering, Faculty of Mechanical Engineering, Cracow University of Technology, Cracow, Poland

Allowable Temperature Rates for Pressure Components Using European Standards

Monitoring of Thermal Stresses in Pressure Components of Steam Boilers

Zhou Wei Advanced Packaging R\&D, Micron Semiconductor Asia Pte Ltd, Singapore, Singapore

Thermal Stresses at Multi-Material Corners and Cracks in Plastic IC Packages

Dieter Weichert Institute of General Mechanics, RWTH Aachen University, Aachen, Germany

Experimental Analysis of Hot Spotting in Sliding Systems

Limit States and Failure of Elastoplastic Structures Under

Thermomechanical Loading 
Noah Weiss Northwestern University, Evanston, IL, USA

Steady State Heat Flow and Isolated Crack

Steady State Heat Flow into Concentrated Contact

V. M. Wheeler Department of Mechanical Engineering, University of Minnesota, Minneapolis, MN, USA

Ballistic-Diffusive Approximation: A New Look

C-and F-Processes Model: A Generalized Approach to Solving Transient Diffusive, Wavelike and Ballistic Solid State Heat Conduction Problems

Equation of Phonon Radiative Transport: Formulation and Analysis by the Weighted Residual Method

David M. Williamson SMF Fracture and Shock Physics Group, Cavendish Laboratory, University of Cambridge, Cambridge, UK

Hot Spot Ignition of Explosives and Their Thermal Properties and

Experimental Measurement of the Thermal Properties of a PBX and Its Binder System

David A. Willis Department of Mechanical Engineering, Southern Methodist University, Dallas, TX, USA

Stress Generation in Laser-Material Interaction: Phase Explosion Phenomenon

Krzysztof Wilmanski Technische Universität Berlin and ROSE School, Pavia, Italy

Thermomechanics of Diffusive Porous and Granular Materials

Alan Wineman Department of Mechanical Engineering, University of Michigan, Ann Arbor, MI, USA

Constitutive Equation for Linear Viscoelastic Materials with

Temperature-Dependent Properties

Sławomir Wiśniewski Dept. of Heat Engineering, West Pomeranian University of Technology, Szczecin, Szczecin, Poland

Transient Heat Conduction in Semi-infinite Solid with Specified Surface Temperature

Tomasz S. Wiśniewski Institute of Heat Engineering, Faculty of Power and Aeronautical Engineering, Warsaw University of Technology, Warsaw, Poland

Thermal Contact Resistance

Transient Heat Conduction in Semi-infinite Solid with Specified Surface Heat Flux

Janusz Wojtkowiak Institute of Environmental Engineering, Poznań University of Technology, Poznań, Poland

Lumped Thermal Capacity Model

Benxin Wu Department of Mechanical, Materials and Aerospace Engineering, Illinois Institute of Technology, Chicago, IL, USA

Thermal Stress in Laser Shock Peening 
Chien-Huei Wu Investech Precision Casting Co., Kaohsiung, Taiwan, Republic of China

Laminated Plates, Antisymmetric and Nonsymmetric

Laminated Plates, Symmetric

Guan-Yuan Wu Department of Fire Science, Central Police University, Taoyuan, Taiwan, Republic of China

Magneto-Thermo-Dynamic Instability of a Beam With Magnetic Fields and Thermal Loads

Jun Xiao College of Material Science and Technology, Nanjing University of Aeronautics and Astronautics, Nanjing, People's Republic of China

Laminated Plates, Interlaminar Stresses

Rui Xiaoting Institute of Launch Dynamics, Nanjing University of Sciences and Technology, Nanjing, People's Republic of China

Aerothermoelastic Behavior of Flat and Curved Panels

Aerothermoelastic Behavior of Lifting Surfaces

Dongji Xie Nvidia Corp, Santa Clara, CA, USA

Pad Cratering and Role of Thermal Stresses

Jun Jiang Xiong School of Transportation Science and Engineering, Beihang University, Beijing, People's Republic of China

Residual Thermal Stress in 2D Orthogonal Plain Weave Fabric

Composites

Su-Ming Xiong College of Electrical Engineering, Zhejiang University, Hangzhou, People's Republic of China

Magneto-electro-thermoelastic Problems: Fundamental Solutions and Green's Function

Thermoelastostatics of Transversely Isotropic Materials: Fundamental Solutions and Green's Functions

Xianfan Xu School of Mechanical Engineering and Birck Nanotechnology Center, Purdue University, West Lafayette, IN, USA

Thermoelastic Waves Induced by Pulsed Laser Heating

Yepeng Xu College of Civil Engineering, Nanjing University of Technology, Nanjing, Jiangsu Province, People's Republic of China Plates of Variable Thickness

Anas H. Yaghi Manufacturing Simulation Division, Manufacturing Technology Centre, Coventry, Coventry, UK

Finite Element Simulation of the Fusion Welding of Metal Components Including Post-weld Heat Treatment

Koji Yamamoto Mitsuboshi Diamond Industrial Co., Ltd, Settsu, Osaka, Japan Glass Scribing (Processing) Methods Using Thermal Stresses Induced by Laser Heating 
Anatoliy Yasinskyy Pidstryhach Institute for Applied Problems of Mechanics and Mathematics, National Academy of Sciences of Ukraine, Lviv, Ukraine Determination and Optimization of Stress State of Bodies on the Basis of Inverse Thermoelasticity Problems

Aleksander Yevtushenko Faculty of Mechanical Engineering, Bialystok University of Technology, Białystok, Poland

FEM-Modeling of Frictional Heating During Braking

Laser-Induced Thermal Splitting in Homogeneous Body with Coating

One-Dimensional Analytical Models of Frictional Heating During

Braking

Sung Yi Mechanical and Material Engineering Department, Portland State University, Portland, OR, USA

Hygrothermally Induced Residual Stresses and Failures in Plastic IC Packages During Reflow Process

Yun-Bo Yi Department of Mechanical and Materials Engineering, University of Denver, Denver, CO, USA

Perturbation Methods in Thermoelastic Instability (TEI) with Finite Element Implementation

Thermoelastic Damping in Resonators

Faruk Yigit Department of Mechatronics Engineering, Yildiz Technical University, Yildiz, Istanbul, Turkey

Thermomechanical Growth Instability in Solidification

Bekir Sami Yilbas Mechanical Engineering Department, King Fahd University of Petroleum and Minerals, Dhahran, Saudi Arabia

Thermal Stress Development in the Solid Due to Laser Pulse Irradiation: Analytical Approaches for Temperature and Stress Fields

Ching-Chung Yin Department of Mechanical Engineering, National Chiao Tung University, Hsinchu, Taiwan, Republic of China

Thermoelastic Waves in Thin Bodies

Chen Yong School of Mechanical Engineering, Shanghai Jiaotong University, Shanghai, People's Republic of China

Body Force Method

Body Force Method for Thermoelasticity

Hamdy M. Youssef Mathematics Department/Faculty of Education, Alexandria University, Alexandria, Egypt

Wave Propagation in the Two-Temperature Theory of

Thermoelasticity

Xiaoguang Yuan Department of Earth and Space Science and Engineering, York University, Toronto, Canada

Theory of Pyroelectrics with Finite Wave Speeds 
Yanan Yue School of Power and Mechanical Engineering, Wuhan University, Wuhan, Hubei, People's Republic of China

Stresses in Laser Surface Nanostructuring

Thermoelastic Waves Induced by Pulsed Laser Heating

Matthew M. F. Yuen Department of Mechanical Engineering, The Hong Kong University of Science and Technology, Kowloon, Hong Kong, People's Republic of China

Thermal Stress in LED Packages for Solid-State Lighting

Andrew V. Zabolotsky "Magnezit Group" ltd., St. Petersburg, Russia Thermal Shock and Modeling of Destruction for Refractory Linings of Metallurgical Installations

Thermal Stress in LED Packages for Solid-State Lighting

Przemyslaw Zagrodzki Raybestos Powertrain, Crawfordsville, IN, USA Clutches, Hot Spotting Behavior

Modal Decomposition Methods in TEI

Arash Zamani Department of Mechanical Engineering, Amirkabir University of Technology, Tehran, Iran

Generalized Thermoelasticity of a Crack Problem Considering

Lord-Shulman Theory

Thermal Fracture by Extended FEM

Vittorio Zampoli Department of Electronic and Computer Science Engineering, University of Salerno, Fisciano, Italy

Asymptotic Partition Backward in Time

A. V. Zemskov State University of Aerospace Technologies, Moscow, Russia

Dynamic Processes in Thermo-Electro-Magneto-Elastic and Thermo-Elasto-Diffusive Media

Method of Asymptotic Separation of Variables in Problems of Thermoelasticity

Method of Averaging in Problems of Thermoelasticity of Composite Materials

Tong-Yi Zhang Department of Mechanical Engineering, Hong Kong University of Science and Technology, Kowloon, Hong Kong, People's Republic of China Fracture of Piezoelectric Materials

Strip Dielectric Breakdown Model in Piezoelectric Fracture Mechanics

Kai Zhang Department of Mechanical Engineering, The Hong Kong University of Science and Technology, Kowloon, Hong Kong, People's Republic of China

Thermal Stress in LED Packages for Solid-State Lighting 
Lu Zhang Link-Belt Construction Equipment Company, Lexington, KY, USA

Welding of Large Structures

MingHao Zhao Henan Key Engineering Laboratory for Anti-fatigue Manufacturing Technology and the School of Mechanical Engineering, Zhengzhou University, Zhengzhou, People's Republic of China

Extended Displacement Discontinuity Boundary Integral Equation Method for Analysis of Cracks in Smart Materials

Nonlinear Fracture Models of Magnetoelectroelastic Media

Yanfei Zhao Department of Civil Engineering, Computer Modeling and Simulation Group, University of Akron, Akron, OH, USA

Cracks in Transversely Isotropic and Inhomogeneous Elastic Solids

Wu Zhen Key Laboratory of Liaoning Province for Composite Structural Analysis of Aerocraft and Simulation, Shenyang Aerospace University, Shenyang, People's Republic of China

High-Order Theory, Laminated Plate Buckling

Laminated Plates, Hygrothermal Effects

Ding Zhou College of Civil Engineering, Nanjing University of Technology, Nanjing, Jiangsu Province, People's Republic of China

Plates of Variable Thickness

Xiangmin Zhou Department of Mechanical Engineering, University of Minnesota, Minneapolis, MN, USA

C- and F-Processes Model and Dynamic Thermoelasticity

Yun Shen Zhou Department of Electrical Engineering, University of Nebraska-Lincoln, Lincoln, NE, USA

Stresses in Laser-Assisted Surface Cleaning

Yichun C. Zhou Key Laboratory of Low Dimensional Materials \& Application Technology, Ministry of Education, Xiangtan University, Hunan, Xiangtan, People's Republic of China

Modeling of Residual Stresses with Thermal Cycling

Wenliang Zhu Ceramic Physics Laboratory \& Research Institute for Nanoscience, Kyoto Institute of Technology, Kyoto, Japan

Raman Spectroscopic Method for Analyzing Residual Stresses in Ceramic Composites

Yaroslav A. Zhuk Department of Thermoelasticity, Timoshenko Institute of Mechanics, Kiev, Ukraine

Dissipative Heating of Thin-Wall Structures Containing Piezoactive

Layers 
Wiesław Zima Institute of Thermal Power Engineering, Faculty of Mechanical Engineering, Cracow University of Technology, Cracow, Poland

Energetics

Multidimensional Heat Conduction Problems with Boundary Conditions of the Third Kind

Space-Marching Method for Two-Dimensional Inverse Heat Conduction Problems

Janusz Zmywaczyk Faculty of Mechatronics and Aerospace, Military University of Technology, Warsaw, Poland

Two-Dimensional, Steady-State Conduction

Two-Dimensional, Steady-State Conduction: Tables

Volodymyr V. Zozulya Centro de Investigacion Cientifica de Yucatan A.C., Mérida, Yucatán, Mexico

Variational Formulation and Nonsmooth Optimization Algorithms in Elastostatic Contact Problems for Cracked Body

Barbara V. Zupančič Center for Experimental Mechanics, Faculty of Mechanical Engineering, University of Ljubljana, Ljubljana, Slovenia Experimental Determination of Material Time-Dependent Properties 



\section{Notations}

In part, the adopted system of notations follows that used by R. B. Hetnarski and J. Ignaczak in The Mathematical Theory of Elasticity, second edition, CRC Press, Boca Raton, 2011. The system, in turn, is based on M. E. Gurtin, The Linear Theory of Elasticity, Encyclopedia of Physics, Chief Editor: S. Flügge,vol. VIa/2, Volume Editor: C. Truesdell, Springer, 1972. In general, scalars are shown as italic light face letters, vectors are denoted by bold lower case letters, second-order tensors and fourth-order tensors appear as bold upper case letters. For example, $\boldsymbol{v}$, velocity vector, is a vector with components $v_{i}, \mathbf{S}$, stress tensor, is a second-order tensor with components $S_{i j}$, and $\mathbf{C}$, elasticity tensor, is a fourth-order tensor with components $C_{i j k l}$. Alternatively, stresses may be written as $\sigma_{i j}$ and strains as $e_{i j}$.

If contributors used the notations as in the list, they did not need to include notation definitions in their entries.

Many contributors, however, used notations of their own, and they defined all symbols in their entries.

\section{List of Symbols}

$A_{i j}, B_{i j}, C_{i j} \quad$ First, second, and third metric tensor of the surface

A Beltrami solution, thermal expansion tensor

$\mathbf{A}(\mathbf{m}) \quad$ Acoustic tensor for a direction $\mathbf{m}$

$B \quad$ Body, Boussinesq's function

C Elasticity tensor

D Torsional rigidity, flexural rigidity, domain, Biot's free energy function

$D_{i} \quad$ Electric displacement component

D Finite strain tensor

E Young's modulus

$E^{3} \quad$ Three-dimensional Euclidean space

$E^{2} \quad$ Two-dimensional Euclidean space

$\mathbf{E}$ or $e_{i j} \quad$ Infinitesimal strain tensor

$\mathbf{E}^{\perp} \quad$ Normal part of $\mathbf{E}$ with respect to a plane

$\mathbf{E}^{\|} \quad$ Tangential part of $\mathbf{E}$ with respect to a plane

$\varepsilon_{E}(\mathbf{E}) \quad$ Strain energy density of a progressive wave

$\varepsilon_{E}\left(\mathbf{E}^{\perp}\right) \quad$ Normal strain energy density of a progressive wave

$\varepsilon_{S}(\mathbf{S}) \quad$ Stress energy density of a progressive wave 
$\varepsilon_{S}\left(\mathbf{S}^{\perp}\right) \quad$ Normal stress energy density of a progressive wave

$\varepsilon_{S}\left(\mathbf{S}^{\|}\right) \quad$ Tangential stress energy density of a progressive wave

$F \quad$ Airy stress function, magnitude of force

F Deformation gradient, force

$G \quad$ Shear modulus, Green's function, gravitatioal energy, Gibbs thermo-dynamical potential

$H(\cdot) \quad$ Heaviside function

H Harmonic second-order tensor field, compatibility related fourth-order tensor

I Moment of inertia of an area, intrinsic energy per unit volume

$I_{1}, I_{2}, I_{3} \quad$ Invariants of stress tensor

$J \quad$ Polar moment of inertia of an area

$K \quad$ Stress concentration factor

$K_{r} \quad$ Displacement concentration factor

$K(t) \quad$ Kinetic energy

K Stiffness matrix

$L \quad$ Length

$L\{f(t)\} \quad$ Laplace transform of $f(t)$

$L^{-1}\{\bar{f}(p)\} \quad$ Inverse Laplace transform of $\bar{f}(p)$

$M \quad$ Bending moment

$M_{\alpha \beta} \quad$ Bending and twisting moments

M Stress-temperature tensor

$N \quad$ Stress resultant

$N_{\alpha \beta} \quad$ Normal and shear forces

0 Origin, zero vector, zero tensor

$P \quad$ Part of $B$, concentrated force

$Q \quad$ Heat supply field, magnitude of shear force

Q Orthogonal tensor

$R \quad$ Region in $E^{3}$; distance between two points, radius in spherical coordinate system, normal radius of curvature in shells, heat produced per unit time per unit volume

$R, \theta, \phi \quad$ Spherical coordinates $\left(\theta\right.$ is in plane $x_{1}, x_{2}$, and $\phi$ is between $x_{3}$ and $R$ )

R Riemann - Christoffel curvature tensor

$S \quad$ Entropy per unit volume; surface traction

$\mathbf{S}$ or $\sigma_{\mathrm{ij}} \quad$ Stress tensor

$\widehat{S}(B) \quad$ Mean stress

$S^{\perp} \quad$ Normal part of $\mathbf{S}$ with respect to a plane

$S^{\|} \quad$ Tangential part of S with respect to a plane

$T \quad$ Absolute temperature, time interval

$T_{0} \quad$ Initial temperature

$U \quad$ Internal energy, generalized strain energy function

$U_{C}\{\mathbf{E}\} \quad$ Strain energy

$u(t) \quad$ Total energy of $B$ at time $t$

$V \quad$ Vector space associated with $E^{3}$

W Work 


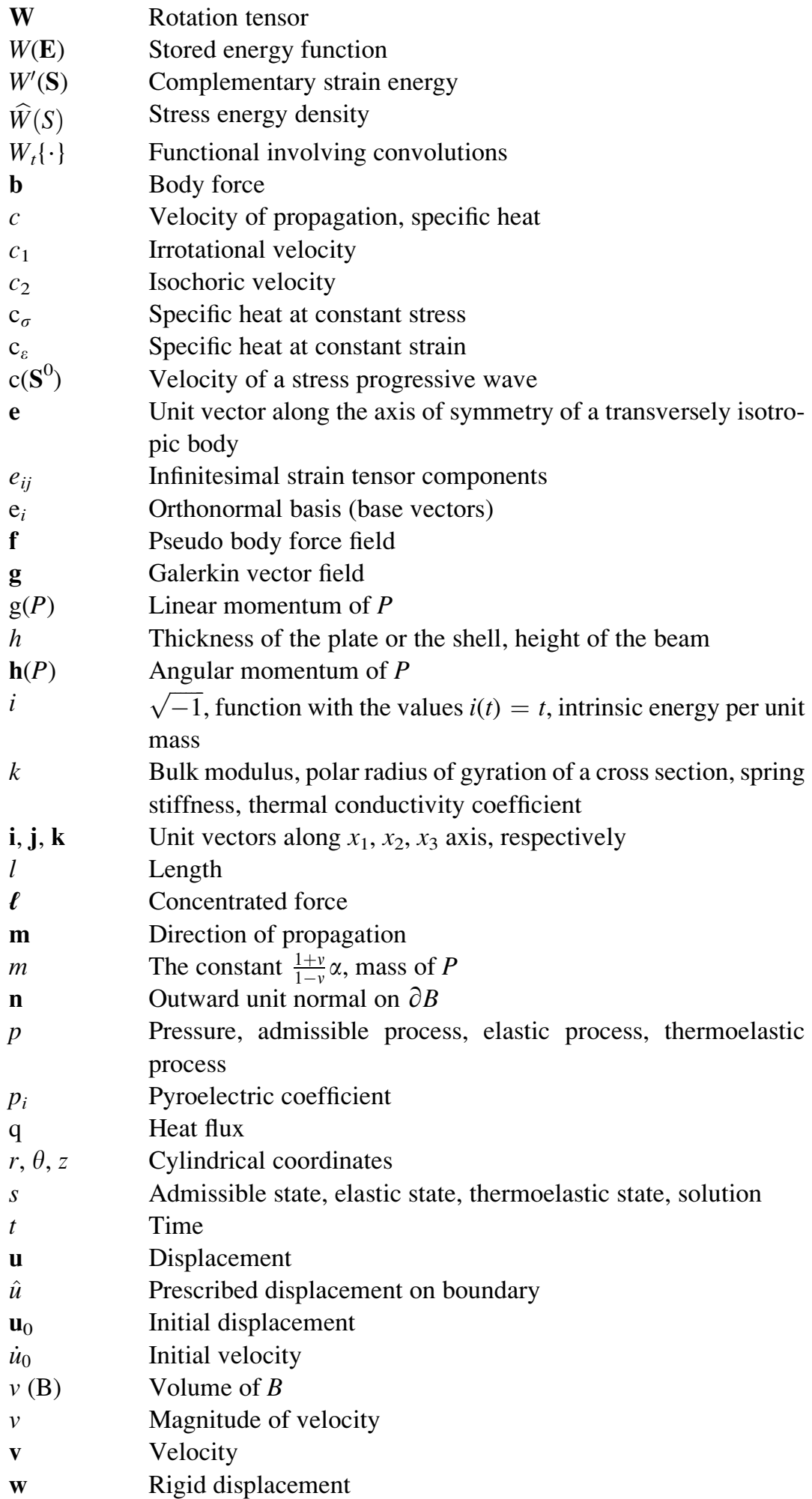




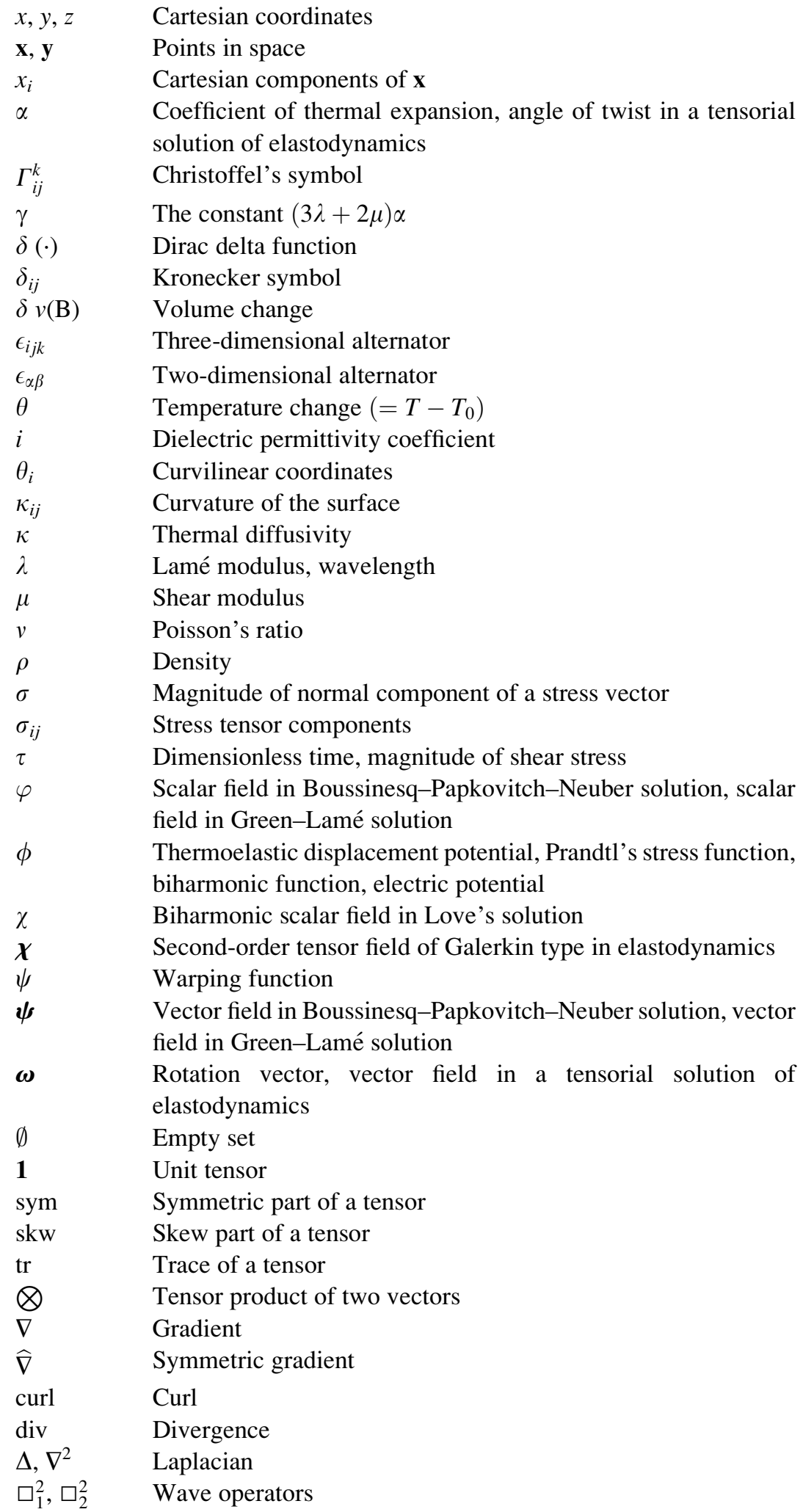


*

$\llbracket \cdot \rrbracket$ $d a$

$d v$

(')

()$^{T}$
Convolution

Jump in a function

Element of area

Element of volume

Time derivative

Transpose of a tensor 